\title{
Planck intermediate results. XIV. Dust emission at millimetre wavelengths in the Galactic plane
}

\author{
Planck Collaboration: P. A. R. Ade ${ }^{78}$, N. Aghanim ${ }^{54}$, M. I. R. Alves ${ }^{54 \star}$, M. Arnaud ${ }^{67}$, M. Ashdown ${ }^{64,6}$, F. Atrio-Barandela ${ }^{18}$, J. Aumont $^{54}$, \\ C. Baccigalupi ${ }^{77}$, A. J. Banday ${ }^{82,10}$, R. B. Barreiro ${ }^{61}$, J. G. Bartlett ${ }^{1,62}$, E. Battaner ${ }^{84}$, K. Benabed ${ }^{55,81}$, A. Benoit-Lévy ${ }^{24,55,81}$, J.-P. Bernard ${ }^{82,10}$, \\ M. Bersanelli ${ }^{32,45}$, P. Bielewicz ${ }^{82,10,77}$, J. Bobin ${ }^{67}$, A. Bonaldi ${ }^{63}$, J. R. Bond ${ }^{9}$, J. Borrill ${ }^{13,79}$, F. R. Bouchet ${ }^{55,81}$, F. Boulanger ${ }^{54}$, M. Bucher ${ }^{1}$, \\ C. Burigana ${ }^{44,30}$, R. C. Butler ${ }^{44}$, J.-F. Cardoso ${ }^{68,1,55}$, A. Catalano ${ }^{69,66}$, A. Chamballu ${ }^{67,15,54}$, H. C. Chiang ${ }^{26,7}$, L.-Y. Chiang ${ }^{57}$, P. R. Christensen ${ }^{74,35}$, \\ D. L. Clements ${ }^{51}$, S. Colombi ${ }^{55,81}$, L. P. L. Colombo ${ }^{23,62}$, F. Couchot ${ }^{65}$, B. P. Crill ${ }^{62,75}$, A. Curto ${ }^{6,61}$, F. Cuttaia ${ }^{44}$, L. Danese ${ }^{77}$, R. D. Davies ${ }^{63}$, \\ R. J. Davis ${ }^{63}$, P. de Bernardis ${ }^{31}$, A. de Rosa ${ }^{44}$, G. de Zotti ${ }^{41,77}$, J. Delabrouille ${ }^{1}$, C. Dickinson ${ }^{63}$, J. M. Diego ${ }^{61}$, H. Dole ${ }^{54,53}$, S. Donzelli ${ }^{45}$, \\ O. Doré ${ }^{62,11}$, M. Douspis ${ }^{54}$, X. Dupac ${ }^{38}$, T. A. Enßlin ${ }^{72}$, H. K. Eriksen ${ }^{59}$, E. Falgarone ${ }^{66}$, F. Finelli ${ }^{44,46}$, O. Forni ${ }^{82,10}$, M. Frailis ${ }^{43}$, E. Franceschi ${ }^{44}$, \\ S. Galeotta ${ }^{43}$, K. Ganga ${ }^{1}$, T. Ghosh ${ }^{54}$, M. Giard ${ }^{82,10}$, G. Giardino ${ }^{39}$, J. González-Nuevo ${ }^{61,77}$, K. M. Górski ${ }^{62,85}$, A. Gregorio ${ }^{33,43,48}$, A. Gruppuso ${ }^{44}$, \\ F. K. Hansen ${ }^{59}$, D. L. Harrison ${ }^{58,64}$, C. Hernández-Monteagudo ${ }^{12,72}$, D. Herranz ${ }^{61}$, S. R. Hildebrandt ${ }^{11}$, E. Hivon ${ }^{55,81}$, W. A. Holmes ${ }^{62}$, \\ A. Hornstrup ${ }^{16}$, W. Hovest ${ }^{72}$, A. H. Jaffe ${ }^{51}$, W. C. Jones ${ }^{26}$, M. Juvela ${ }^{25}$, E. Keihänen ${ }^{25}$, R. Keskitalo ${ }^{21,13}$, T. S. Kisner ${ }^{71}$, R. Kneiss1 ${ }^{37,8}$, \\ J. Knoche ${ }^{72}$, M. Kunz ${ }^{17,54,3}$, H. Kurki-Suonio ${ }^{25,40}$, G. Lagache ${ }^{54}$, A. Lähteenmäki ${ }^{2,40}$, J.-M. Lamarre ${ }^{66}$, A. Lasenby ${ }^{6,64}$, R. J. Laureijs ${ }^{39}$,

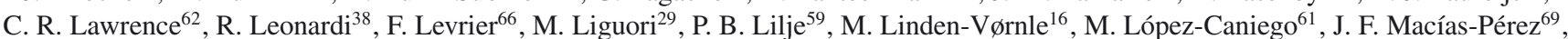 \\ B. Maffei ${ }^{63}$, D. Maino ${ }^{32,45}$, N. Mandolesi ${ }^{44,5,30}$, M. Maris ${ }^{43}$, D. J. Marshall ${ }^{67}$, P. G. Martin ${ }^{9}$, E. Martínez-González ${ }^{61}$, S. Masi ${ }^{31}$, S. Matarrese ${ }^{29}$, \\ P. Mazzotta ${ }^{34}$, A. Melchiorri ${ }^{31,47}$, L. Mendes ${ }^{38}$, A. Mennella ${ }^{32,45}$, M. Migliaccio ${ }^{58,64}$, S. Mitra ${ }^{50,62}$, M.-A. Miville-Deschênes ${ }^{54,9}$, A. Moneti ${ }^{55}$, \\ L. Montier ${ }^{82,10}$, G. Morgante ${ }^{44}$, D. Mortlock ${ }^{51}$, D. Munshi ${ }^{78}$, J. A. Murphy ${ }^{73}$, P. Naselsky ${ }^{74,35}$, F. Nati ${ }^{31}$, P. Natoli ${ }^{30,4,44}$, H. U. Nørgaard-Nielsen ${ }^{16}$, \\ F. Noviello ${ }^{63}$, D. Novikov ${ }^{51}$, I. Novikov ${ }^{74}$, C. A. Oxborrow ${ }^{16}$, L. Pagano ${ }^{31,47}$, F. Pajot ${ }^{54}$, R. Paladini ${ }^{52}$, D. Paoletti ${ }^{44,46}$, F. Pasian ${ }^{43}$, G. Patanchon ${ }^{1}$, \\ M. Peel ${ }^{63}$, O. Perdereau ${ }^{65}$, F. Perrotta ${ }^{77}$, F. Piacentini ${ }^{31}$, M. Piat ${ }^{1}$, E. Pierpaoli2 ${ }^{23}$, D. Pietrobon ${ }^{62}$, S. Plaszczynski ${ }^{65}$, E. Pointecouteau ${ }^{82,10}$, \\ G. Polenta ${ }^{4,42}$, N. Ponthieu ${ }^{54,49}$, L. Popa ${ }^{56}$, G. W. Pratt ${ }^{67}$, S. Prunet ${ }^{55,81}$, J.-L. Puget ${ }^{54}$, J. P. Rachen ${ }^{20,72}$, W. T. Reach ${ }^{83}$, R. Rebolo ${ }^{60,14,36}$, \\ M. Reinecke ${ }^{72}$, M. Remazeilles ${ }^{63,54,1}$, C. Renault ${ }^{69}$, S. Ricciardi ${ }^{44}$, T. Riller ${ }^{72}$, I. Ristorcelli ${ }^{82,10}$, G. Rocha ${ }^{62,11}$, C. Rosset ${ }^{1}$,
}

J. A. Rubiño-Martín ${ }^{60,36}$, B. Rusholme ${ }^{52}$, M. Sandri ${ }^{44}$, G. Savini ${ }^{76}$, D. Scott ${ }^{22}$, L. D. Spencer ${ }^{78}$, J.-L. Starck ${ }^{67}$, V. Stolyarov ${ }^{6,64,80}$, F. Sureau ${ }^{67}$, D. Sutton ${ }^{58,64}$, A.-S. Suur-Uski ${ }^{25,40}$, J.-F. Sygnet ${ }^{55}$, J. A. Tauber $^{39}$, D. Tavagnacco ${ }^{43,33}$, L. Terenzi ${ }^{44}$, L. Toffolatti ${ }^{19,61}$, M. Tomasi $^{45}$, M. Tristram $^{65}$, M. Tucci ${ }^{17,65}$, L. Valenziano ${ }^{44}$, J. Valiviita ${ }^{40,25,59}$, B. Van Tent $^{70}$, L. Verstraete ${ }^{54}$, P. Vielva $^{61}$, F. Villa ${ }^{44}$, N. Vittorio ${ }^{34}$, L. A. Wade $^{62}$, B. D. Wandelt ${ }^{55,81,28}$, D. Yvon ${ }^{15}$, A. Zacchei ${ }^{43}$, and A. Zonca ${ }^{27}$

(Affiliations can be found after the references)

Received 25 July 2013 / Accepted 28 January 2014

\begin{abstract}
We use Planck HFI data combined with ancillary radio data to study the emissivity index of the interstellar dust emission in the frequency range $100-353 \mathrm{GHz}$, or $3-0.8 \mathrm{~mm}$, in the Galactic plane. We analyse the region $l=20^{\circ}-44^{\circ}$ and $|b| \leq 4^{\circ}$ where the free-free emission can be estimated from radio recombination line data. We fit the spectra at each sky pixel with a modified blackbody model and two opacity spectral indices, $\beta_{\mathrm{mm}}$ and $\beta_{\mathrm{FIR}}$, below and above $353 \mathrm{GHz}$, respectively. We find that $\beta_{\mathrm{mm}}$ is smaller than $\beta_{\mathrm{FIR}}$, and we detect a correlation between this low frequency power-law index and the dust optical depth at $353 \mathrm{GHz}, \tau_{353}$. The opacity spectral index $\beta_{\mathrm{mm}}$ increases from about 1.54 in the more diffuse regions of the Galactic disk, $|b|=3^{\circ}-4^{\circ}$ and $\tau_{353} \sim 5 \times 10^{-5}$, to about 1.66 in the densest regions with an optical depth of more than one order of magnitude higher. We associate this correlation with an evolution of the dust emissivity related to the fraction of molecular gas along the line of sight. This translates into $\beta_{\mathrm{mm}} \sim 1.54$ for a medium that is mostly atomic and $\beta_{\mathrm{mm}} \sim 1.66$ when the medium is dominated by molecular gas. We find that both the two-level system model and magnetic dipole emission by ferromagnetic particles can explain the results. These results improve our understanding of the physics of interstellar dust and lead towards a complete model of the dust spectrum of the Milky Way from far-infrared to millimetre wavelengths.
\end{abstract}

Key words. ISM: general - Galaxy: general - radiation mechanisms: general - radio continuum: ISM - submillimeter: ISM

\section{Introduction}

The frequency coverage of Planck ${ }^{1}$ is opening new windows in our understanding of Galactic emission. This is especially the case for the high frequency data that provide an all-sky view

^ Corresponding author: M. I. R. Alves e-mail: marta.alves@ias.u-psud.fr

1 Planck (http://www.esa.int/Planck) is a project of the European Space Agency (ESA) with instruments provided by two scientific consortia funded by ESA member states (in particular the lead countries France and Italy), with contributions from NASA (USA) and telescope reflectors provided by a collaboration between ESA and a scientific consortium led and funded by Denmark. of the Rayleigh-Jeans regime of the thermal dust spectrum. The emission at the Planck high frequency bands $(350 \mu \mathrm{m}-3 \mathrm{~mm})$ is dominated by the contribution of big grains (radius larger than $0.05 \mu \mathrm{m}$ ), which heated by stellar photons are in thermal equilibrium with the interstellar radiation field (ISRF). The spectral energy distribution (SED) of big dust grains is usually approximated by a modified blackbody emission law of the form

$I_{v}=\tau_{v_{0}}\left(\frac{v}{v_{0}}\right)^{\beta} B_{v}\left(v, T_{\mathrm{d}}\right)$,

where $\tau_{v_{0}}$ is the dust optical depth at a reference frequency $v_{0}$, $\beta$ the spectral index of the opacity, and $B_{v}$ the Planck function, 
which depends on both the frequency and the dust temperature $T_{\mathrm{d}}$. However, early observations by the Cosmic Background Explorer (COBE) indicated that the modified blackbody spectrum does not provide a good description of the dust SED from far-infrared (FIR) to millimetre wavelengths (Reach et al. 1995). Later works have confirmed that $\beta$ appears to vary with frequency, the SED flattening in the millimetre relative to the best single modified blackbody fit and also varying with environment (Finkbeiner et al. 1999; Galliano et al. 2005; Paladini et al. 2007; Planck Collaboration XVII 2011; Planck Collaboration XIX 2011). Studies of dust analogues (e.g., Agladze et al. 1996; Boudet et al. 2005; Coupeaud et al. 2011) have characterized the FIR and millimetre emission of different types of amorphous silicates. These show a frequency, as well as temperature, dependence of $\beta$ not unlike the astronomical results. The astrophysical interpretation of this flattening is under study as new observations become available, and some possible explanations have been suggested. One possibility is a description of the opacity of the big grains in terms of a two-level system (TLS, Meny et al. 2007). Alternatively, it might be attributed to magnetic dipole emission from magnetic particles (Draine \& Hensley 2013) or to the evolution of carbon dust (Jones et al. 2013).

To study the low frequency flattening of the dust SED in the Galactic plane, we need to take the free-free emission from the ionized gas into account. Free-free emission is a principal foreground contaminant of the cosmic microwave background (CMB), not only at radio frequencies, where it is comparable to other Galactic components such as synchrotron, but also at millimetre wavelengths where the thermal dust emission dominates. It becomes a major component in the Galactic plane where it is produced by the gas ionized by recently formed massive stars. All-sky maps of the free-free emission, derived in the context of CMB foreground studies, have been obtained directly from $\mathrm{H} \alpha$ measurements (Dickinson et al. 2003; Finkbeiner 2003). However, this optical line suffers from large dust absorption along the Galactic plane, and thus fails to provide a reliable measure of the thermal emission at low Galactic latitudes. A freefree map that includes the Galactic plane is essential, not only to correctly evaluate the CMB power spectrum at low angular frequency, but also for Galactic star formation studies. The WMAP satellite has provided all-sky maps at five microwave frequencies that have been combined to estimate the contribution of freefree, synchrotron, thermal dust and anomalous microwave emission (AME) using a maximum entropy method (MEM, Bennett et al. 2013). Another approach using hydrogen radio recombination lines (RRLs) has been presented recently by Alves et al. (2010, 2012). In contrast to $\mathrm{H} \alpha$, these radio lines at a frequency of $1.4 \mathrm{GHz}$ are optically thin and are not absorbed by dust or the radio emitting plasma. The RRL method has provided the first direct measure of the diffuse free-free emission along the Galactic plane, in the longitude range $l=20^{\circ}-44^{\circ}$ and for latitudes $|b| \leq 4^{\circ}$ (Alves et al. 2012).

The free-free emission has a spectral index $\alpha \equiv \mathrm{d} \ln \left(I_{v}\right) /$ $\mathrm{d} \ln v$, varying from -0.10 at $1.4 \mathrm{GHz}$ to -0.15 at $100 \mathrm{GHz}$. The free-free emission dominates at frequencies between 60 and $100 \mathrm{GHz}$, but there the other Galactic components, namely synchrotron, dust and AME, also contribute to the total intensity. The AME is an additional Galactic component observed in the frequency range $10-60 \mathrm{GHz}$ (e.g., Kogut et al. 1996; Leitch et al. 1997; de Oliveira-Costa et al. 1997; Planck Collaboration XX 2011; Planck Collaboration Int. XII 2013; Planck Collaboration Int. XV 2014) which cannot be explained by free-free, synchrotron or thermal dust emission and is thought to arise from small spinning dust grains (Draine \& Lazarian 1998; Ali-Haïmoud et al. 2009; Ysard \& Verstraete 2010; Hoang et al. 2010, 2011). In a spectral decomposition of the four Galactic emission components along the plane, Planck Collaboration Int. XXII (in prep.) find that the AME contribution is comparable to that of the free-free in the frequency range 20-40 GHz. On the other hand, and due to its steeper spectral index, $-1.2 \lesssim \alpha \lesssim-0.7$ (Davies et al. 1996; Ghosh et al. 2012), the synchrotron emission is mostly dominant at frequencies less than a few gigahertz.

This paper aims to characterize for the first time the dust emissivity in the frequency range $100-353 \mathrm{GHz}$ of the diffuse emission in the Galactic plane. For this purpose, we remove the free-free emission contribution using the RRL data (Sect. 3.1). We start by describing the Planck and ancillary data used in this work in Sects. 2 and 3. In Sect. 4 we present the data analysis techniques, followed by the main results of the paper in Sect. 5 . These are discussed further and interpreted in Sect. 6, followed by the conclusions in Sect. 7.

\section{Planck HFI data}

Planck (Tauber et al. 2010; Planck Collaboration I 2011; Planck Collaboration Int. XXII, in prep.) is the third-generation space mission to measure the anisotropy of the CMB. It observed the sky in nine frequency bands covering $28.5-857 \mathrm{GHz}$ with high sensitivity and angular resolution from 32.24 to 4.33 . The Low Frequency Instrument (LFI; Mandolesi et al. 2010; Bersanelli et al. 2010; Mennella et al. 2011; Planck Collaboration II 2014) covered the $28.4,44.1$, and $70.4 \mathrm{GHz}$ bands with amplifiers cooled to $20 \mathrm{~K}$. The High Frequency Instrument (HFI; Lamarre et al. 2010; Planck HFI Core Team 2011; Planck Collaboration VI 2014) covered the 100, 143, 217, 353, 545, and $857 \mathrm{GHz}$ bands with bolometers cooled to $0.1 \mathrm{~K}$. Polarization is measured in all but the highest two bands (Leahy et al. 2010; Rosset et al. 2010).

In the present work we use data from the Planck 2013 data release which can be obtained from the Planck Legacy Archive $^{2}$. We use the HFI data acquired between 13 August 2009 and 27 November 2010. These are converted to intensity units of $\mathrm{MJy} \mathrm{sr}^{-1}$ following the IRAS SED convention (Beichman et al. 1988), which assumes a spectral index $\alpha=-1$. Colour corrections based on the observed emission spectrum and on the spectral response of the receiver, are applied to derive the specific intensity at the effective frequency of each band (Planck Collaboration IX 2014). The Planck CMB map is derived from the SMICA component separation method and presented in Planck Collaboration XII (2014). Close to the plane of the Galaxy, it is not possible to correctly separate the CMB fluctuations from the much brighter Galactic emission. Hence, this region of the SMICA map has been replaced by a constrained realization of the CMB fluctuations. For this reason we derive our results using the data uncorrected for the CMB fluctuations, which we compare with those obtained when the SMICA CMB map is subtracted from the data (Sect. 5.4). The lowest HFI frequency band also has the lowest angular resolution, of 9.65. However we smooth the Planck data to a common resolution of $15^{\prime}$, assuming Gaussian beams, to match the lower resolution of the RRL data (Sect. 3.1).

The $100 \mathrm{GHz}$ data are significantly contaminated by the $\mathrm{CO} J=1 \rightarrow 0$ line at $115 \mathrm{GHz}$ and the $217 \mathrm{GHz}$ data by the

\footnotetext{
2 http://www.sciops.esa.int/index.php? project=planck\&page=Planck_Legacy_Archive
} 
CO $J=2 \rightarrow 1$ line at $230 \mathrm{GHz}$. At $353 \mathrm{GHz}$ the contribution of the $\mathrm{CO} J=3 \rightarrow 2$ line is small, but not negligible compared to dust emission. The CO line emission is subtracted using the Planck TYPE $1 \mathrm{CO}$ maps from the MILCA (Modified Independent Linear Combination Algorithm, Hurier et al. 2013) bolometer solution (Planck Collaboration XIII 2014). These are converted from line integrated units to intensity units as described in Planck Collaboration XIII (2014). The calibration uncertainties on these maps are of 10,2 , and $5 \%$ at 100 , 217, and $353 \mathrm{GHz}$, respectively. The $100 \mathrm{GHz}$ MILCA map has been compared with ground-based data, in particular the Dame et al. (2001) ${ }^{12} \mathrm{CO} J=1 \rightarrow 0$ survey along the Galactic plane, for which there is an overall agreement of $16 \%$ (Planck Collaboration XIII 2014). However, in the Galactic plane region of the present study, both datasets agree within $25 \%$. This discrepancy can be explained by the shifting of the $\mathrm{CO}$ line frequency due to Doppler effects, that is to say, the rotation of the Galactic disk (Planck Collaboration XIII 2014).

The overall calibration uncertainties for the Planck HFI maps are $10 \%$ at 857 and $545 \mathrm{GHz}, 1.2 \%$ at $353 \mathrm{GHz}$, and $0.5 \%$ at lower frequencies. These values are increased at the lowest frequencies due to the subtraction of the $\mathrm{CO}$ and free-free emission. We did not subtract the zodiacal dust emission from the maps, because it is a negligible contribution in the Galactic plane (Planck Collaboration XIV 2014). Moreover, the cosmic infrared background (CIB) monopole was removed from all the HFI maps as described in Planck Collaboration XI (2014).

\section{Ancillary data}

Along with Planck HFI we need to use ancillary data, namely RRL observations for the removal of the free-free emission and IRAS data to constrain the dust temperature. All data sets are in HEALPix format (Górski et al. 2005), at $N_{\text {side }}=512$, and are smoothed to a common resolution of $15^{\prime}$.

\subsection{Radio Recombination Line data}

A fully-sampled map of the free-free emission in the Galactic plane region $l=20^{\circ}-44^{\circ}$ and $|b| \leq 4^{\circ}$ has been derived by Alves et al. (2012) using RRL data. These data are from the H I Parkes All-Sky Survey and associated Zone of Avoidance Survey (Staveley-Smith et al. 1996, 1998) at $1.4 \mathrm{GHz}$ and $15^{\prime}$ resolution. One source of uncertainty on these data is the conversion from the observed antenna temperature to intensity units, which requires a detailed knowledge of the observing beam (Rohlfs \& Wilson 2000). The RRL data presented in Alves et al. may need a correction downwards of $5-10 \%$, since they were converted to a scale appropriate for point sources; this correction depends on the angular size of the source relative to the main beam of $15^{\prime}$. The free-free brightness temperature ${ }^{3}$ estimated from the RRL integrated line emission depends on the electron temperature of the ionized gas as $T_{\mathrm{e}}^{1.15}$ (Gordon \& Sorochenko 2009). Alves et al. used an average value of $T_{\mathrm{e}}=6000 \mathrm{~K}$; an increase of this value by $500 \mathrm{~K}(1000 \mathrm{~K})$ would increase the brightness temperature by $10 \%(19 \%)$.

The RRL free-free data are similarly used in the work of Planck Collaboration Int. XXII (in prep.) to separate the different emission components in the Galactic plane and to determine the contribution of the AME. In that work, the free-free map estimated from the radio data are compared to two other free-free

\footnotetext{
3 Following the definition of brightness temperature by Spitzer (1978), in the Rayleigh-Jeans regime.
}

solutions, given by the Planck fastMEM (Planck Collaboration Int. XXII, in prep.) and WMAP MEM (Bennett et al. 2013) component separation methods. The fastMEM and WMAP results agree within $2 \%$ but they are about $20 \%$ higher than the RRL estimation. The proposed solution to this difference is to scale the free-free map from Alves et al. (2012) upwards by 10\%, which is equivalent to increasing the electron temperature to $7000 \mathrm{~K}$ (Planck Collaboration Int. XXII, in prep.). In this paper we use the same electron temperature of $7000 \mathrm{~K}$ and adopt an overall calibration uncertainty of $10 \%$ in the free-free continuum estimated from the RRL data.

\subsection{IRAS data}

We use the IRIS (Improved Reprocessing of the IRAS survey) data at $100 \mu \mathrm{m}$ (Miville-Deschênes \& Lagache 2005) to constrain the peak of the thermal dust emission. The calibration uncertainty for these data is $13.5 \%$.

\subsection{HI data}

The HI data from the Galactic All-Sky Survey (GASS, McClure-Griffiths et al. 2009) are used to estimate the column density of the atomic medium. The GASS survey mapped the 21-cm line emission in the southern sky, $\delta<1^{\circ}$, at $14 ! 4$ angular resolution and $1 \mathrm{~km} \mathrm{~s}^{-1}$ velocity resolution. We use the data corrected for instrumental effects, stray radiation and radio frequency interference from Kalberla et al. (2010). The average temperature uncertainties for these data are below $1 \%$. The H I line is integrated as described in Planck Collaboration Int. XVII (2014) and converted to H I column density assuming that the line is optically thin. The optically thin limit is a simplistic approach in the Galactic plane and results in an underestimation of the true column density, by about $30-50 \%$ as found in H I continuum absorption studies (Strasser \& Taylor 2004).

\section{Analysis}

The aim here is to determine the power-law index of the interstellar dust opacity at the lowest HFI frequencies, which we do by fitting the dust SED.

As mentioned in Sect. 1, $\beta$ appears to be frequency dependent with a break observed at frequencies around $600 \mathrm{GHz}$, or $\lambda \sim 500 \mu \mathrm{m}$ (Paradis et al. 2009; Gordon et al. 2010; Galliano et al. 2011). Planck Collaboration XXV (2011) also found that a single modified blackbody curve accurately fits the FIR spectrum of Galactic molecular clouds, but leaves large residuals at frequencies below $353 \mathrm{GHz}$. For this reason we decided to fit the dust SED using a modified blackbody model, but allowing $\beta$ to vary with frequency, having $\beta=\beta_{\text {FIR }}$ for $v \geq 353 \mathrm{GHz}$ and $\beta=\beta_{\mathrm{mm}}$ for $v<353 \mathrm{GHz}$. Using the Planck HFI bands along with the IRAS $100 \mu \mathrm{m}$ data, we also solve for the other parameters in Eq. (1), namely $T_{\mathrm{d}}$ and $\tau_{353}$, where we take the reference frequency as $353 \mathrm{GHz}$ for the dust optical depth.

The Planck maps at frequencies above $353 \mathrm{GHz}$ contain mainly dust emission and also CIB emission. The CIB fluctuations have a power spectrum flatter than that of the interstellar dust (Miville-Deschênes et al. 2002; Lagache et al. 2007; Planck Collaboration XVIII 2011), thus contributing mostly at small angular scales and producing a statistically homogeneous signal. This signal only represents a significant fraction of the total brightness in the most diffuse high latitude regions of the sky, and thus can be neglected in the Galactic plane.

In the range $100-353 \mathrm{GHz}$, even though most of the emission comes from interstellar dust, both the CMB and free-free 


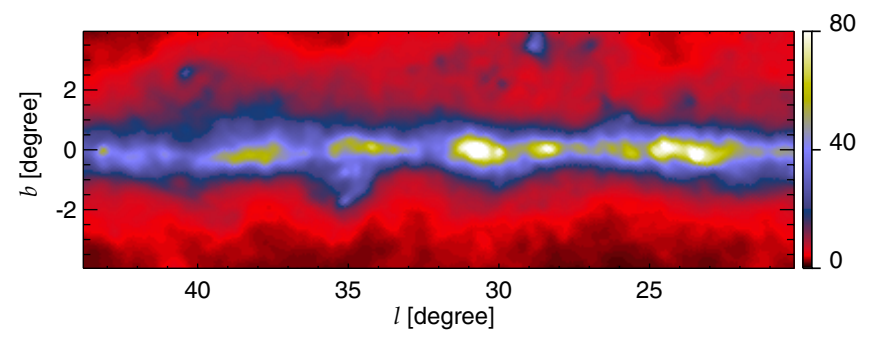

Fig. 1. HFI $353 \mathrm{GHz}$ map of the Galactic plane region $l=20^{\circ}-44^{\circ}$, $|b| \leq 4^{\circ}$, in units of $\mathrm{MJy} \mathrm{sr}^{-1}$ and at $15^{\prime}$ resolution.

components also contribute to the total brightness. The fluctuations of the CMB are faint, rms of about $80 \mu \mathrm{K}$ at scales of $15^{\prime}$, compared to the brightest emission in the Galactic plane. Therefore, we can neglect the contribution from the CMB fluctuations, since its rms temperature is about $5 \%$ of the total emission in the thin disk of the Galaxy. However, at latitudes $|b| \gtrsim 2^{\circ}$ the $\mathrm{CMB}$ fluctuations at $100 \mathrm{GHz}$ are about 10 times brighter than the free-free emission. The effects of neglecting the CMB component at these higher latitudes will be investigated via simulations in Sect. 5.3, as well as using the SMICA CMB map in Sect. 5.4.

At $|b| \lesssim 1^{\circ}$, the contribution of the free-free emission can be as high as $20-40 \%$ to the total emission at $100 \mathrm{GHz}$, from both the diffuse and the individual H II regions. Therefore, we need to remove the free-free emission if we are to fit the dust spectrum only with a modified blackbody model. For this purpose, we use the free-free map estimated from the RRLs (Sect. 3.1), as this is currently the only direct measure of this emission in the Galactic plane, in particular in the $24^{\circ} \times 8^{\circ}$ region centred on $(l, b)=\left(32^{\circ}, 0^{\circ}\right)$. This region is shown in Fig. 1, in the Planck $353 \mathrm{GHz}$ channel. The free-free continuum, estimated from the RRL data at $1.4 \mathrm{GHz}$, is extrapolated to the HFI frequencies using a frequency dependent Gaunt factor (Eq. (10.9) of Draine 2011) and an electron temperature of $7000 \mathrm{~K}$.

We used the IDL MPFIT routine to fit the final SEDs pixelby-pixel in the $24^{\circ} \times 8^{\circ}$ region. This routine performs weighted least-squares fitting of the data (Markwardt 2009), taking into account the noise (both statistical noise and systematic uncertainties) for each spectral band. We also include a noise term from the CMB fluctuations, typically $80 \mu \mathrm{K}$, which will be dominant outside the Galactic plane and at the lowest frequencies. These uncertainties are used to give weights to the spectral points. Colour corrections based on the local spectral index across each band were applied to both Planck and IRAS data during the model-fitting procedure (Planck Collaboration IX 2014).

\section{The dust spectral index from FIR to millimetre wavelengths}

In this section we present the main results of this work, namely the difference between $\beta_{\mathrm{FIR}}$ and $\beta_{\mathrm{mm}}$ in the Galactic plane and how the latter relates to changes in dust temperature and optical depth. Several tests are performed to assess the robustness of the results, including a validation of the analysis techniques via simulations.

\subsection{Flattening of the dust SED}

The spectra at the position of a complex of $\mathrm{H}$ II regions, $\mathrm{G} 24.5+0.0$ (W42), and towards a diffuse region in the Galactic plane centred at $(l, b)=(40.5,0.0)$ are shown in Fig. 2. The

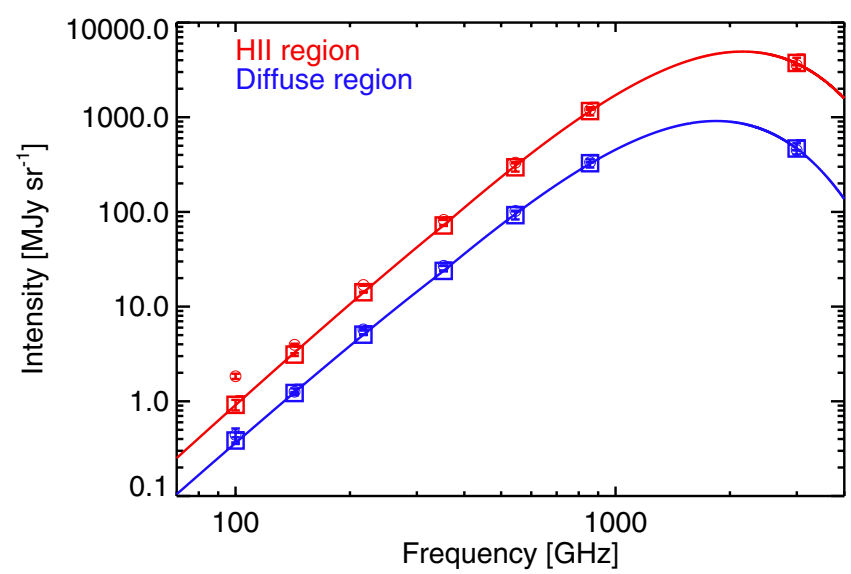

Fig. 2. Spectra towards the H II region complex W42 (red) and a diffuse region in the Galactic plane centred at $(l, b)=(40.5,0 \circ 0)$ (blue). The circles show the total intensity (corrected for CO emission) and the squares show the same data after subtraction of the free-free contribution. All the data points are shown with their corresponding uncertainties.

fitted models are also shown. The effect of subtracting the free-free emission is clearly visible at $100 \mathrm{GHz}$ in the spectrum of the H II region (compare the circles with the squares); at frequencies above $143 \mathrm{GHz}$ this subtraction is negligible. The spectral indices of the $\mathrm{H}$ II region are $\beta_{\mathrm{FIR}}=1.9 \pm 0.2$ and $\beta_{\mathrm{mm}}=1.7 \pm 0.1$, while for the diffuse region, $\beta_{\mathrm{FIR}}=1.9 \pm 0.2$ and $\beta_{\mathrm{mm}}=1.6 \pm 0.1$. These values suggest that the diffuse region has a flatter millimetre spectrum than the H II region. The uncertainties on the parameters reflect the likely mixture of dust components along the line of sight, which have a range of temperatures and different properties. The $\chi^{2}$ values of the fits are 3.1 and 1.1, for the HII region and the diffuse region respectively, with $^{4} N_{\text {d.o.f. }}=3$. The $\chi^{2} / N_{\text {d.o.f. }}$ values across the map are usually lower than one, meaning that the fits are within the uncertainties of each point. The uncertainties on the data at frequencies of $217 \mathrm{GHz}$ and above are dominated by the calibration uncertainties, which are correlated across the channels. At these frequencies, the median value of our fit residuals across the map is close to zero and within the overall uncertainties of the data, thus indicating that the fits are a good representation of the data. At 100 and $143 \mathrm{GHz}$ the histograms of the percentage residual emission are centred at $2 \%$ and $-3 \%$, respectively. These values are higher than the $0.5 \%$ calibration uncertainty at these frequencies but lower than the final uncertainties once the noise contribution from $\mathrm{CMB}$ and the uncertainties associated with free-free and $\mathrm{CO}$ templates are included.

The distributions of temperature and spectral indices fitted for the $24^{\circ} \times 8^{\circ}$ region under study are shown in Fig. 3 . The dust temperature ranges from 16 to $24 \mathrm{~K}$, with a median value of $19 \mathrm{~K}$. Even though we are describing the SED with only a single temperature whilst a range of temperatures are expected along the line of sight especially in the Galactic plane, the higher temperature regions found here are associated with $\mathrm{H}$ II regions, as expected from local heating by their OB stars. Similarly, colder regions are associated with molecular clouds.

The histograms of $\beta_{\mathrm{FIR}}$ and $\beta_{\mathrm{mm}}$ are compared in Fig. $3 \mathrm{~b}$ for the whole $24^{\circ} \times 8^{\circ}$ region. The $\beta_{\text {FIR }}$ distribution has a median value of 1.76 and a standard deviation $(\sigma$, corresponding to the $68.3 \%$ confidence interval) of 0.08 and that of $\beta_{\mathrm{mm}}$ has a median value of 1.55 with $\sigma=0.12$. This indicates that the $\beta_{\mathrm{mm}}$ distribution is centred at a lower value and is also broader.

4 Degrees of freedom (d.o.f. $=N_{\text {points }}-N_{\text {parameters }}$ ). 
Planck Collaboration: Dust emission at millimetre wavelengths in the Galactic plane
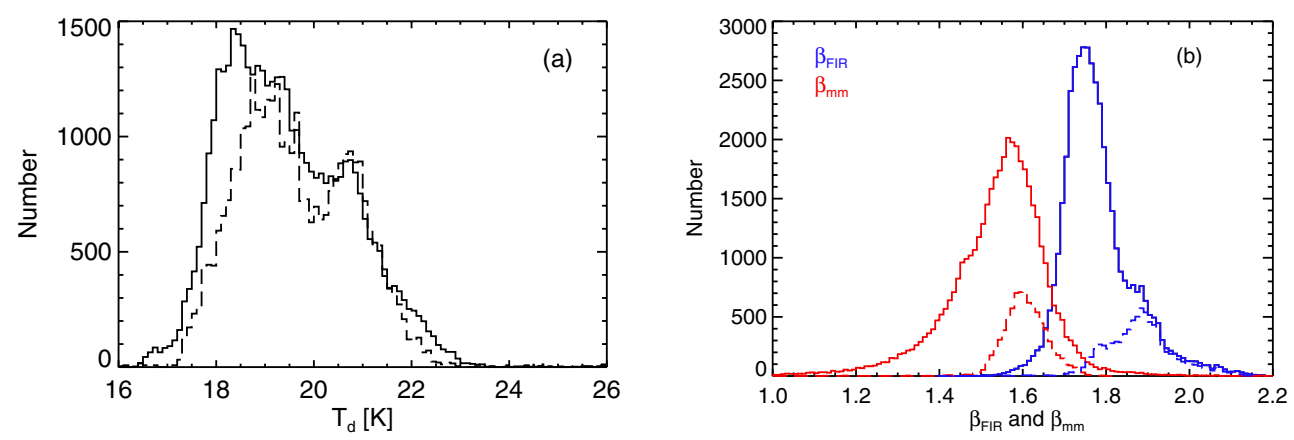

Fig. 3. Histograms of the dust temperature a) and dust opacity indices b) for the $24^{\circ} \times 8^{\circ}$ region. The dashed lines in both panels correspond to the pixels where $\tau_{353} \geq 4 \times 10^{-4}$. In panel a) the dashed histogram is scaled up by a factor of four.
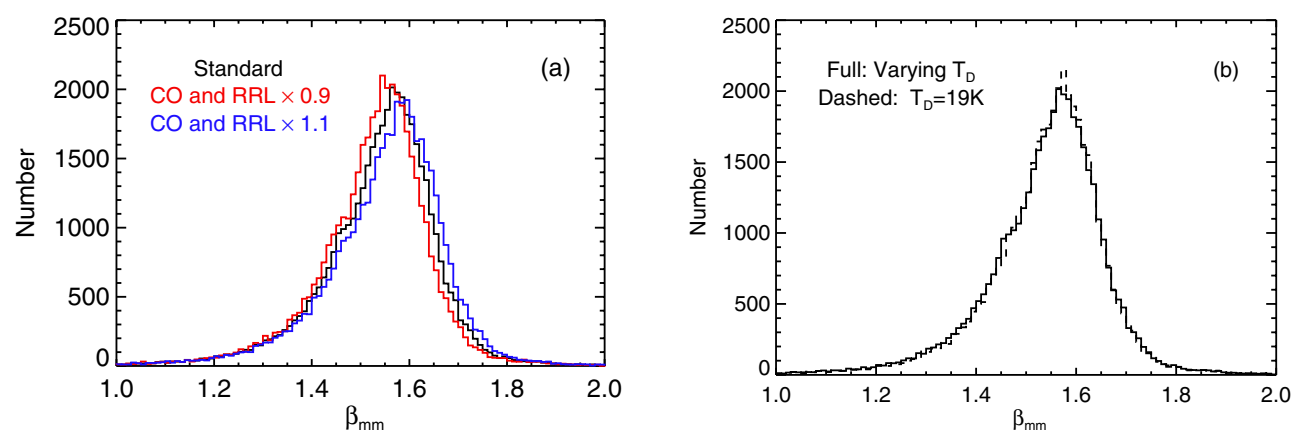

Fig. 4. Comparison of the results on $\beta_{\mathrm{mm}}$ a) when the $\mathrm{CO}$ and free-free corrections vary by $10 \%$; b) when $T_{\mathrm{d}}$ is allowed to vary in the fit and also when $T_{\mathrm{d}}$ is fixed to a single value of $19 \mathrm{~K}$.

If we select the pixels within $|b| \lesssim 1^{\circ}$, which represents regions with an optical depth $\tau_{353} \geq 4 \times 10^{-4}$, the corresponding $\beta_{\mathrm{FIR}}$ and $\beta_{\mathrm{mm}}$ histograms, shown as dashed lines, have median values of $1.88, \sigma=0.08$, and $1.60, \sigma=0.06$ respectively. The shift in the mean values of both $\beta_{\mathrm{mm}}$ and $\beta_{\mathrm{FIR}}$ is related to a variation of these parameters from the diffuse to the denser medium, as will be discussed in Sect. 6.2. The $\beta_{\mathrm{mm}}$ values fitted outside the narrow Galactic plane are affected by CMB fluctuations, which become brighter than the free-free and are not taken into account in the fit. The impact of the CMB in the $\beta_{\mathrm{mm}}$ results will be further analysed in Sects. 5.3 and 5.4. The dust temperature distribution is similar between the $24^{\circ} \times 8^{\circ}$ region and the thin Galactic disk (full and dashed lines in Fig. 3a), with a sharper decrease of the latter below $19 \mathrm{~K}$.

The histogram of $\beta_{\mathrm{FIR}}$ in Fig. 3 does not include the effects of the calibration uncertainties, namely its width only takes into account the variations across the map. This is an important point when assessing the difference between $\beta_{\mathrm{FIR}}$ and $\beta_{\mathrm{mm}}$, as given by Fig. 3. At frequencies of $353 \mathrm{GHz}$ and above, where the contribution of $\mathrm{CO}$, free-free and $\mathrm{CMB}$ are negligible compared to dust emission, the data uncertainties are dominated by calibration uncertainties. We performed Monte Carlo simulations to estimate this effect on $\beta_{\mathrm{FIR}}$ and found that, in 1000 simulations, the dispersion around an input value of 1.75 is 0.17 . This value is about twice that measured from the $\beta_{\text {FIR }}$ histograms of Fig. 3. Nevertheless, in the thin Galactic disk, this does not affect the difference measured between $\beta_{\mathrm{FIR}}$ and $\beta_{\mathrm{mm}}$. A further check on the quality of the SED fits and the importance of including a second spectral index, $\beta_{\mathrm{mm}}$, is given by comparing the residuals with those resulting from a model with a single $\beta$. When only one spectral index is fit for from 100 to $3000 \mathrm{GHz}$, the median value of the residuals across the map is larger at all frequencies, relative to the two- $\beta$ model. In particular, the median value of the residuals at $857 \mathrm{GHz}, 11 \%$, is higher than the calibration uncertainty. We also note that, if we choose the reference frequency of $545 \mathrm{GHz}$, instead of $353 \mathrm{GHz}$ for the break in the spectral index, the fits also result in larger residuals at all frequencies.

We tested the robustness of the fitted $\beta_{\mathrm{mm}}$ against calibration uncertainties in both the $\mathrm{CO}$ and the free-free templates by varying the subtraction of the CO and RRL contributions, at all frequencies, by $10 \%$ (Sects. 2 and 3.1). An under-subtraction of either the $\mathrm{CO}$ or the free-free emission could in principle result in a lower $\beta_{\mathrm{mm}}$. However, as Fig. 4a illustrates, $\beta_{\mathrm{mm}}$ is essentially insensitive to these variations. This is due to the fact that dust is the dominant emission component at these frequencies, combined with the higher uncertainties of the data at 143 and $100 \mathrm{GHz}$.

In order to investigate the impact of the dust temperature on $\beta_{\mathrm{mm}}$, we compare the results when $T_{\mathrm{d}}$ is fixed to $19 \mathrm{~K}$ with those when $T_{\mathrm{d}}$ is allowed to vary in the SED fit. These are shown in Fig. $4 \mathrm{~b}$, where it is seen that the distribution of $\beta_{\mathrm{mm}}$ is unaffected when using a constant or varying value of $T_{\mathrm{d}}$ across the region.

\subsection{Variations with temperature and optical depth}

An anti-correlation between $\beta$ and $T_{\mathrm{d}}$ has been detected in previous observations in a variety of Galactic regions (Dupac et al. 2003; Désert et al. 2008; Paradis et al. 2010; Planck Collaboration XXV 2011). This seems to indicate that the dust opacity index decreases with temperature, even if part of this effect can be attributed to data noise and to temperature mixing along the line of sight (Sajina et al. 2006; Shetty et al. 2009b,a; Juvela \& Ysard 2012b,a). Kelly et al. (2012) show that the former can be mitigated by using a hierarchical Bayesian technique. The distribution of $\beta_{\text {FIR }}$ as a function of $T_{\mathrm{d}}$ is shown in Fig. 5a, 

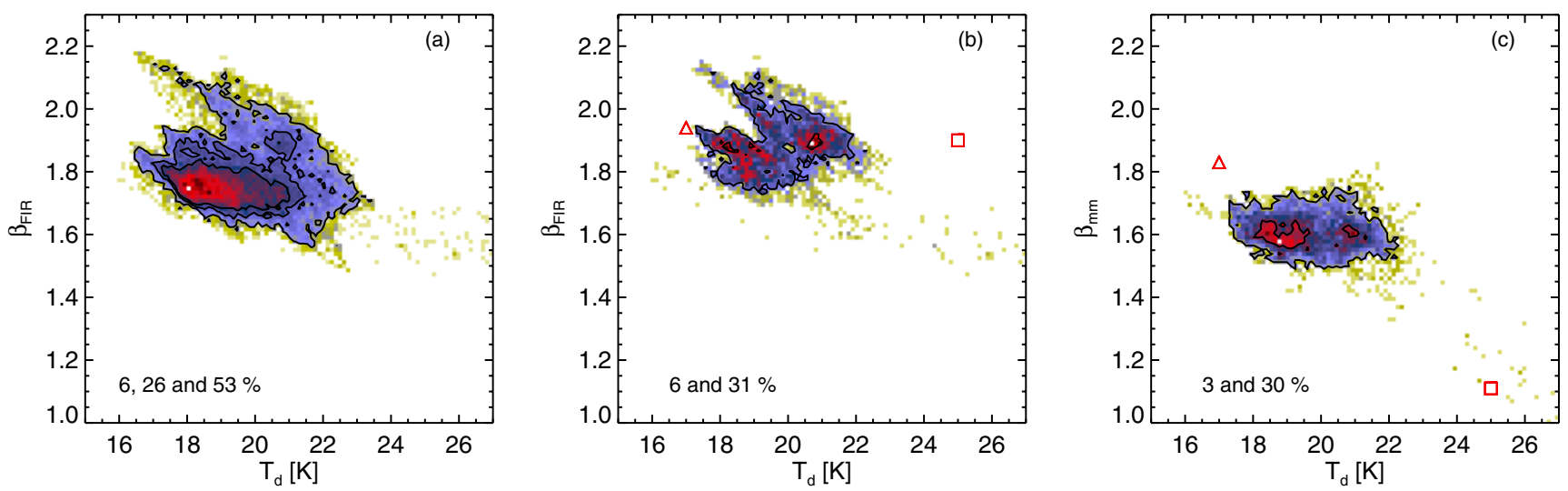

Fig. 5. Dust spectral indices as a function of temperature. a) $\beta_{\text {FIR }}$ versus $T_{\mathrm{d}}$ for the whole $24^{\circ} \times 8^{\circ}$ region. b) $\beta_{\text {FIR }}$ versus $T_{\mathrm{d}}$ for points where $\tau_{353} \geq 4 \times 10^{-4}$. c) $\beta_{\mathrm{mm}}$ versus $T_{\mathrm{d}}$ for points where $\tau_{353} \geq 4 \times 10^{-4}$. The triangle and square in panels b) and c) indicate the values obtained by fitting the emissivities predicted by the TLS model (Paradis et al. 2011) for $T_{\mathrm{d}}=17$ and $25 \mathrm{~K}$ (see Sect. 6.1.2). The colour scale is logarithmic and it represents the density of points. The contours show the densities for the cumulated fractions, given by the values in each panel, of the data points, from red to yellow.

for all the points in the $24^{\circ} \times 8^{\circ}$ region. The Pearson correlation coefficient ${ }^{5}$ between the uncertainties on these two parameters is around -0.95 across the map. However, such a strong anticorrelation is not observed in Fig. 5a, nor in Fig. 5b, where $\beta_{\text {FIR }}-T_{\mathrm{d}}$ is plotted for the thin Galactic disk, $|b| \lesssim 1^{\circ}$. Therefore, there is a real variation of $\beta_{\text {FIR }}$ across this region which decreases the anti-correlation trend generated by the data noise.

The distribution of $\beta_{\mathrm{mm}}$ as a function of $T_{\mathrm{d}}$, for the thin Galactic disk, where $\tau_{353} \geq 4 \times 10^{-4}$, is shown in Fig. 5 c. For this region, the correlation coefficient between the uncertainties on $\beta_{\mathrm{mm}}$ and $T_{\mathrm{d}}$ varies between -0.06 and -0.03 . A value of -0.5 is reached outside the Galactic disk, where the signal-to-noise ratio decreases due to the CMB noise term included in the data uncertainties. Thus, Fig. $5 \mathrm{c}$ indicates that there is no evident trend of $\beta_{\mathrm{mm}}$ with $T_{\mathrm{d}}$ (as it is also seen by comparing the corresponding maps in Fig. 8). We note that the range of temperatures that we are probing is limited, about $6 \mathrm{~K}$, which may be due to temperature mixing along the line of sight and local temperature increases around the heating sources present in the Galactic plane.

We find a correlation between $\beta_{\mathrm{mm}}$ and the optical depth $\tau_{353}$, which is shown in Fig. 6. We note that $\beta_{\mathrm{mm}}$ is an intrinsic parameter related to the physics of dust while $\tau_{353}$ scales with the column density of interstellar matter. In Sect. 6 we will describe this empirical correlation in terms of the type of matter rather than the quantity of matter along the line of sight, given by $\tau_{353}$. The scatter in $\beta_{\mathrm{mm}}$ at low optical depth values, or $|b| \gtrsim 1^{\circ}$, is due to the $\mathrm{CMB}$, as discussed above. For $\tau_{353} \geq 4 \times 10^{-4}, \beta_{\mathrm{mm}}$ increases in the highest optical depth regions, as foreshadowed by the results of Fig. 2, which showed an increase of $\beta_{\mathrm{mm}}$ from the diffuse to the H II region. A linear fit to the data, for $\tau_{353} \geq 4 \times 10^{-4}$, gives $\beta_{\mathrm{mm}}=(1.52 \pm 0.01)+(128 \pm 2) \times \tau_{353}$, where we have used the IDL routine regress to perform the linear regression fit, including only the errors on $\beta_{\mathrm{mm}}$. We note that the errors on $\tau_{353}$ are much lower than those on $\beta_{\mathrm{mm}}$. Moreover, the Pearson correlation coefficient between the uncertainties on these two parameters for $\tau_{353} \geq 4 \times 10^{-4}$, varies between 0.03 and 0.08 . This indicates that it is unlikely that the correlation observed is due to data noise.

\footnotetext{
5 The correlation matrix is computed from the covariance matrix of the fit; it measures the intrinsic correlation between the uncertainties on the fit parameters.
}

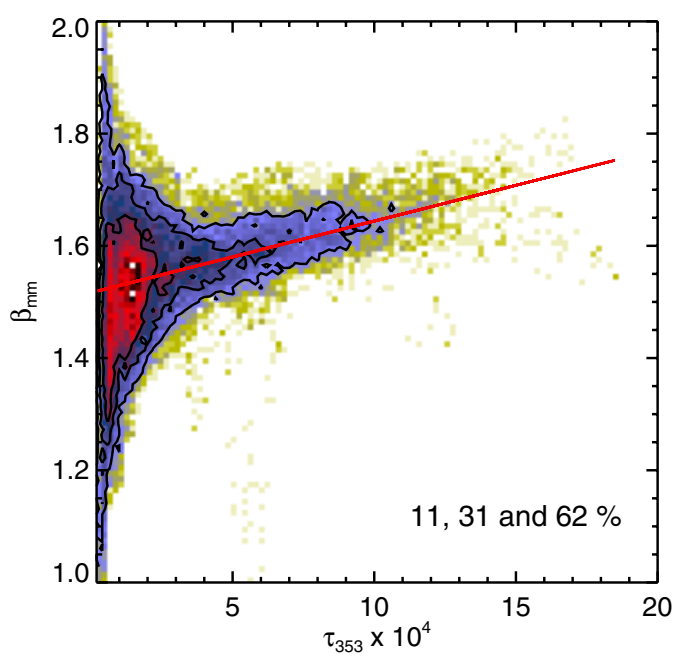

Fig. 6. Distribution of $\beta_{\mathrm{mm}}$ as a function of $\tau_{353}$, for the whole region. The red line gives the best linear fit for $\tau_{353} \geq 4 \times 10^{-4}$ (see text). The colour scale is logarithmic and it represents the density of points. The three contours show the densities for a cumulated fraction of 11,31 , and $62 \%$ of the data points, from red to yellow.

The uncertainty on $\beta_{\mathrm{mm}}$ is statistical; including the systematic uncertainties introduced by the $\mathrm{CMB}, \mathrm{CO}$ and free-free components, which are presented in Sect. 5.3, the correlation is

$\beta_{\mathrm{mm}}=(1.52 \pm 0.02)+(128 \pm 24) \times \tau_{353}$.

\subsection{Validation with simulations}

In order to test the robustness of our fitting procedure against possible biases on $\beta_{\mathrm{mm}}$ associated with the separation of dust emission from $\mathrm{CMB}$, free-free and $\mathrm{CO}$, we apply our routine to simulated maps.

The first simulated maps include dust emission and CMB. We fix $T_{\mathrm{d}}$ to $19 \mathrm{~K}, \beta_{\mathrm{FIR}}$ to 1.75 and $\beta_{\mathrm{mm}}$ to 1.52 across the region. The distribution of $\tau_{353}$ is that obtained from the fit to the data. We reproduce the dust maps at each frequency with a modified blackbody law and add them to the CMB map, reproduced from the best-fit $\Lambda$ CDM model. We then apply the SED fitting routine and recover $\beta_{\mathrm{mm}}$ as a function of the input optical depth as shown in Fig. 7a. A linear fit to the points with $\tau_{353} \geq 4 \times 10^{-4}$ gives 
Planck Collaboration: Dust emission at millimetre wavelengths in the Galactic plane
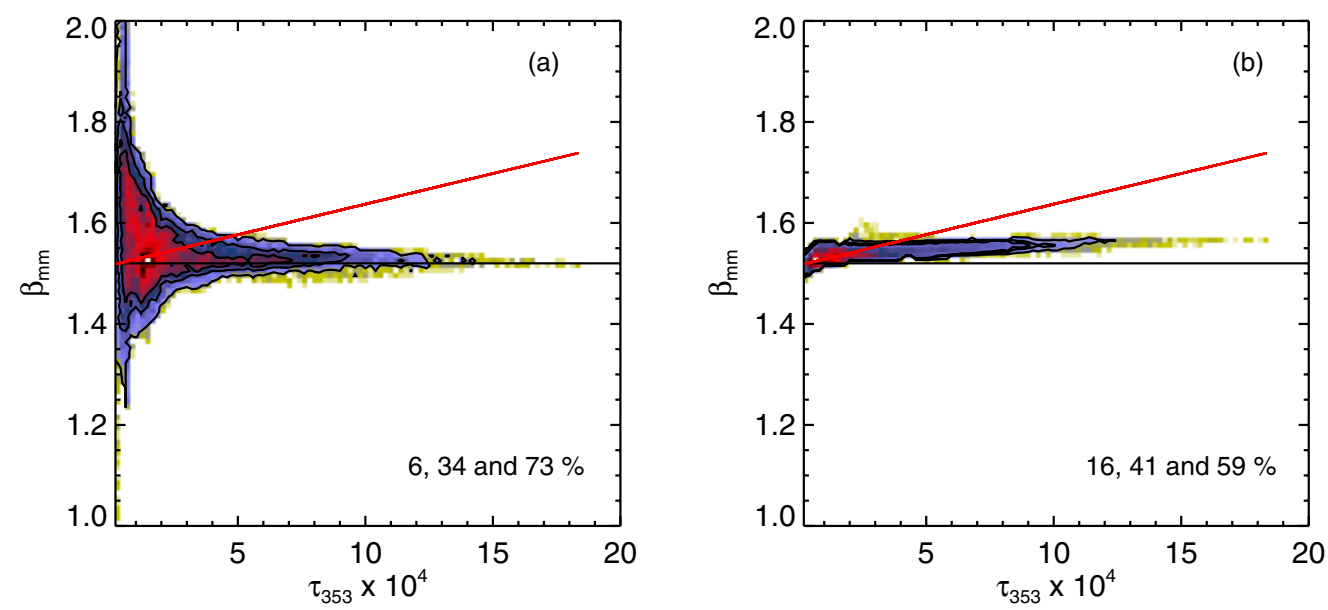

Fig. 7. Dust opacity index $\beta_{\mathrm{mm}}$ recovered from the simulated maps, as a function of the input $\tau_{353}$. The simulated maps in a) have dust emission and $\mathrm{CMB}$, and in b) consist of dust, free-free and CO emission. The black line shows the input $\beta_{\mathrm{mm}}$ of 1.52 in each case; the red lines give the $\beta_{\mathrm{mm}}-\tau_{353}$ relationship derived in Sect. 5. The colour scale is logarithmic and it represents the density of points. The three contours show the densities for the cumulated fractions, given by the values in each panel, of the data points, from red to yellow.

$\beta_{\mathrm{mm}}=1.53-8 \times \tau_{353}$. As discussed in Sect. 5 , the scatter on the $\beta_{\mathrm{mm}}$ values for $\tau_{353} \lesssim 4 \times 10^{-4}$ is created by the CMB when this component is not taken into account in the fit. Moreover, even though it is a minor contributor in the Galactic disk, the CMB also affects the results for higher values of $\tau_{353}$, broadening the $\beta_{\mathrm{mm}}$ distribution around the input value of 1.52 by 0.01 . Thus this result shows that if there is no intrinsic correlation between these two parameters only a limited correlation will be detected. More importantly, Fig. 7a shows that the CMB is not responsible for the $\beta_{\mathrm{mm}}-\tau_{353}$ correlation derived in the previous section.

We also tested our results for a possible bias introduced by incorrect subtraction of $\mathrm{CO}$ and free-free emission from simulated dust maps produced as described above. For that we use the MILCA CO maps (Sect. 2) and subtract $10 \%$ of their emission at 100, 217, and $353 \mathrm{GHz}$. Similarly we remove $10 \%$ of the RRL free-free emission from the simulated dust maps at all frequencies. Such a correction steepens the dust spectrum, as we can see from the results of Fig. 7b. A linear fit to the points gives $\beta_{\mathrm{mm}}=1.53+22 \times \tau_{353}$. This is not, however, capable of reproducing the much steeper slope of $\beta_{\mathrm{mm}}$ with the dust optical depth. For that to be the case, both the CO and RRL maps would have to be systematically underestimated by $30 \%$.

We thus conclude that neither the uncertainty in the $\mathrm{CO}$ and free-free correction of the maps nor omitting the CMB in the spectral fits is responsible for the correlation of $\beta_{\mathrm{mm}}$ with $\tau_{353}$. Finally, Fig. 8 shows the map of $\beta_{\mathrm{mm}}$, estimated using Eq. (2), which presents the same structure as the map of $\tau_{353}$. On the other hand, there is no apparent correlation between the maps of $\beta_{\mathrm{mm}}$ and $T_{\mathrm{d}}$, as it was discussed in Sect. 5.2.

\subsection{Using the Planck SMICA CMB map}

In this section we compare our results with those obtained when the SMICA CMB map is subtracted from each channel map before fitting the dust spectra with a modified blackbody. The resulting $\beta_{\mathrm{mm}}$ as a function of $\tau_{353}$ is shown in Fig. 9. As expected, the scatter on $\beta_{\mathrm{mm}}$ at low values of $\tau_{353}$ decreases, due to the subtraction of the $\mathrm{CMB}$ from the total emission. A linear fit to the points with $\tau_{353} \geq 4 \times 10^{-4}$ gives $\beta_{\mathrm{mm}}=(1.54 \pm 0.01)+$ $(116 \pm 1) \times \tau_{353}$, consistent with Eq. (2). This confirms that the CMB fluctuations, here measured with the SMICA solution, are
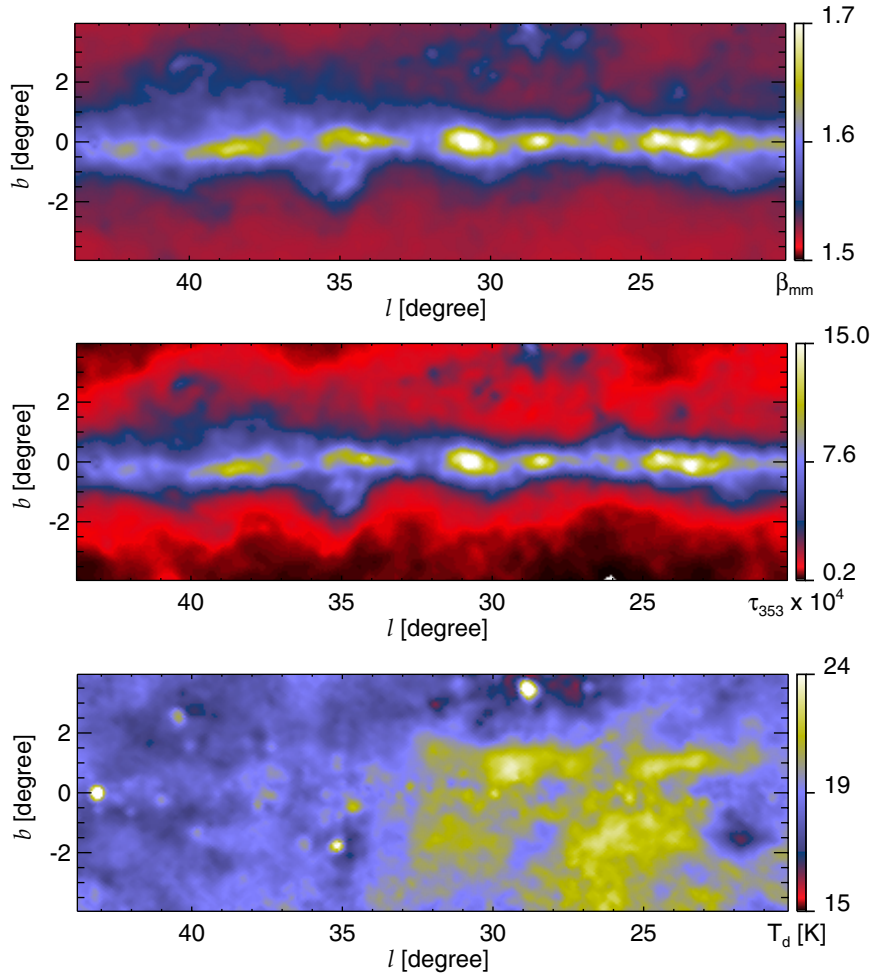

Fig. 8. Maps of the dust parameters. Top: $\beta_{\mathrm{mm}}$, which results from the best linear fit to the correlation with optical depth given by Eq. (2); middle: $\tau_{353}$; bottom: $T_{\mathrm{d}}$.

indeed a small contribution in the Galactic disk and do not affect the main results of the work.

\section{Towards a physical interpretation of the millimetre dust emission}

In this section we compare our results with predictions from current dust models and interpret the empirical relation found between $\beta_{\mathrm{mm}}$ and $\tau_{353}$. 


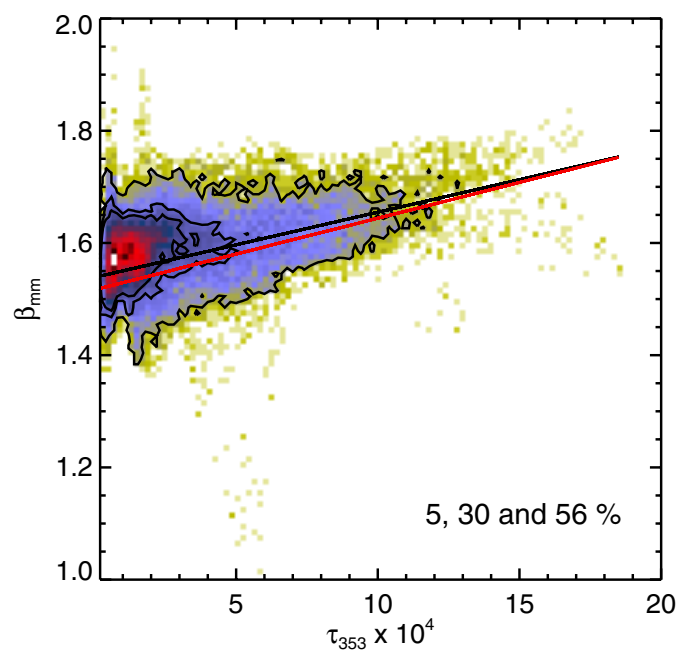

Fig. 9. Distribution of $\beta_{\mathrm{mm}}$ as a function of $\tau_{353}$, for the whole region, when the SMICA CMB map is subtracted from each channel map. The black line gives the $\beta_{\mathrm{mm}}-\tau_{353}$ relationship derived from a fit to the points where $\tau_{353} \geq 4 \times 10^{-4}$, compared to that estimated in Sect. 5 and given by the red line. The colour scale is logarithmic and it represents the density of points. The three contours show the densities for a cumulated fraction of 5,30, and 56\% of the data points, from red to yellow.

\subsection{Dust models}

\subsubsection{Silicate-carbon models}

We start by comparing our results with the predictions of two commonly used dust models, DL07 (Draine \& Li 2007) and DustEM (Compiègne et al. 2011). In particular, we want to investigate whether such models, with two populations of grains dominating the emission at long wavelengths, can explain the flattening of the dust spectrum detected in the present work. Both models use the same optical properties for silicates, for which the opacity scales as $v^{1.6}$, for $\lambda \gtrsim 250 \mu \mathrm{m}$ or $v \lesssim 1200 \mathrm{GHz}$. For the carbon grains DL07 uses the optical properties of graphite, with a spectral index of 2, whereas DustEM uses the laboratory measurements of amorphous carbon, for which the spectral index is 1.6. We use both models to predict the emission in the photometric bands considered in this work, namely IRAS $100 \mu \mathrm{m}$ and HFI, taking the standard size distribution for the diffuse Galactic emission. In order to reproduce conditions closer to those in the Galactic plane, we generate the SEDs for $G_{0}$ values of 1,2 and 4, where $G_{0}$ is the scaling applied to the standard ISRF of Mathis et al. (1983). We then fit the spectra in the same way as the data, namely with a modified blackbody law and two spectral indices, $\beta_{\mathrm{FIR}}$ and $\beta_{\mathrm{mm}}$. The results are shown in Table 1 . First we note that when the radiation field is higher, the peak of the SED is moved to higher frequencies, where the opacity spectral index of silicate grains is larger than 1.6. This can explain the slight increase in $\beta_{\text {FIR }}$ with $G_{0}$. The results also show that $\beta_{\mathrm{mm}}$ is lower than $\beta_{\mathrm{FIR}}$, for both models. Moreover, $\beta_{\mathrm{mm}}$ does not seem to vary with $G_{0}$ or with $T_{\mathrm{d}}$. We find that such models, including two dust components with different opacities can in principle explain the flattening of the dust emission, even if the $\beta$ values recovered from their spectra are lower than those measured from the data.

\subsubsection{Two-level system}

The TLS (Meny et al. 2007) model has been proposed to explain the flattening of the dust emission and its evolution with
Table 1. Results from a modified blackbody fit to the DL07 and DustEM spectra for different scalings of the ISRF, given by $G_{0}$.

\begin{tabular}{lcccccccc}
\hline \hline & \multicolumn{3}{c}{ DL07 } & & \multicolumn{3}{c}{ DustEM } \\
\cline { 2 - 4 } \cline { 7 - 9 }$G_{0}$ & $T_{\mathrm{d}}[\mathrm{K}]$ & $\beta_{\mathrm{FIR}}$ & $\beta_{\mathrm{mm}}$ & & $T_{\mathrm{d}}[\mathrm{K}]$ & $\beta_{\text {FIR }}$ & $\beta_{\mathrm{mm}}$ \\
\hline $1 \ldots \ldots \ldots$ & 21.8 & 1.65 & 1.43 & & 20.1 & 1.58 & 1.48 \\
$\ldots \ldots \ldots$ & 24.1 & 1.70 & 1.43 & & 22.4 & 1.62 & 1.47 \\
$4 \ldots \ldots \ldots$ & 26.7 & 1.73 & 1.43 & & 25.0 & 1.64 & 1.46 \\
\hline
\end{tabular}

temperature. This model consists of three mechanisms which describe the interaction of electromagnetic waves with an amorphous solid. These are temperature-dependent and important in the sub-millimetre, for the range of temperatures relevant to this work. Paradis et al. (2011) use the TLS model to fit the spectrum of the diffuse Galactic emission as well as the spectra of the Archeops sources (Désert et al. 2008). Within this model, the opacity spectral index decreases with increasing temperature. We compare the emissivities predicted by TLS and given in Paradis et al. (2011) with our results for the relevant photometric bands. In particular, we select two spectra, with $T_{\mathrm{d}}$ of 17 and $25 \mathrm{~K}$, within the range of temperatures probed in the present work. We apply our fitting routine to the TLS SEDs to recover $\beta_{\mathrm{FIR}}$ and $\beta_{\mathrm{mm}}$, which are shown in Figs. $5 \mathrm{~b}$ and $\mathrm{c}$. The resulting $\beta_{\text {FIR }}$ values are within the range found in this work, showing a small variation with temperature. However, that is not the case for $\beta_{\mathrm{mm}}$. The values predicted by the TLS model are not within the range of values found in the Galactic disk, and show a steep dependence with temperature. We note that the TLS emissivities used here were computed for a given set of parameters, derived from the combined fit of the diffuse medium emission and the SEDs of the Archeops compact sources (third set of parameters in Table 4 of Paradis et al. 2011). Moreover, they are derived for a single grain, rather than for a grain size distribution. We could argue that the dust temperature estimated from the modified blackbody fit used here is not comparable with that derived from the TLS model. However Paradis et al. (2012) show that both temperatures agree up to about $25 \mathrm{~K}$. Still, we note that $T_{\mathrm{d}}$ obtained from their modified blackbody fit assumes $\beta_{\text {FIR }}=2$. We conclude that the TLS model predicts variations of $\beta_{\mathrm{mm}}$ which are not apparent in the data. We note, however, that the range of temperatures sampled by the data is limited and that if spatial variations of the TLS amplitude, related to the amorphous structure of the grains, were allowed they could easily hide the temperature dependence of $\beta_{\mathrm{mm}}$ in the data.

\subsubsection{Magnetic dipole emission}

The dust emission of the Small Magellanic Cloud (SMC) shows a pronounced flattening towards millimetre wavelengths (Israel et al. 2010; Bot et al. 2010; Planck Collaboration XVII 2011), which, as proposed by Draine \& Hensley (2012), can be explained by magnetic dipole emission from metallic particles. In this section we test the magnetic dipole emission model with the Galactic plane data, which also show excess emission at millimetre wavelengths, even if not as substantial as that observed in the SMC. According to the model of Draine \& Hensley (2012), the iron missing from the gas phase can be locked up in solid grains, either as inclusions in larger grains, in which case they are at the same temperature as the other dust in the diffuse interstellar medium (ISM), $T_{\mathrm{d}} \approx 18 \mathrm{~K}$, or as free-flying nanoparticles, which then have a higher temperature, $T_{\mathrm{d}} \approx 40 \mathrm{~K}$. The emission spectrum of these particles above a resonance frequency, 
Planck Collaboration: Dust emission at millimetre wavelengths in the Galactic plane

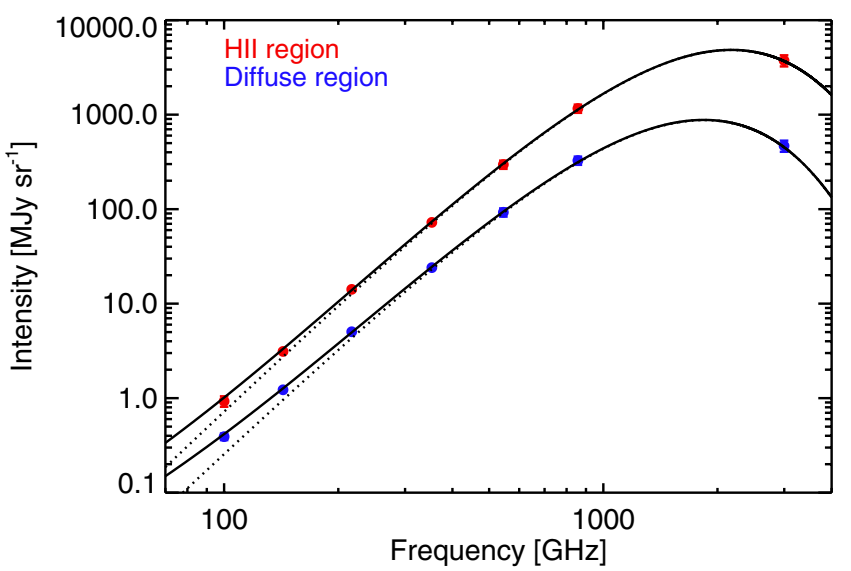

Fig. 10. Spectra towards the H II region complex W42 (red) and a diffuse region in the Galactic plane centred at $(l, b)=\left(40^{\circ} .5,0.0\right)$ (blue). The circles show the total intensity, with their corresponding uncertainties. The dotted lines represent the modified blackbody model, where one single opacity spectral index is fitted to IRAS $100 \mu \mathrm{m}$ and HFI 857, 545 , and $353 \mathrm{GHz}$ data. The solid lines represent the total emission, including the contribution from metallic dust particles (Draine \& Hensley 2013), at the same temperature $T_{\mathrm{d}}$. This contribution to the total emission at $100 \mathrm{GHz}$ is $41 \%$ and $63 \%$ for the HII and diffuse regions, respectively.

$v \sim 15 \mathrm{GHz}$, and below $353 \mathrm{GHz}$, is close to that of a blackbody. In order to test this model, we fit the dust SEDs in the region under study with a modified blackbody of a single opacity in$\operatorname{dex} \beta_{\text {FIR }}$. Its value is determined using the IRAS and HFI 857, 545 , and $353 \mathrm{GHz}$ points and then used to extrapolate the emission to lower frequencies. We include a blackbody spectrum, at the same temperature $T_{\mathrm{d}}$, to represent the metallic particles as inclusions in larger grains, which will account for the excess emission. We find that, at $100 \mathrm{GHz}$, the ratio between the emission from the iron dust particles and that from the modified blackbody, $r_{100}$, has a median of $63 \%$ across the thin Galactic disk, with a standard deviation of $24 \%$. The spectra of the same $\mathrm{HII}$ and diffuse regions as in Fig. 2 are shown in Fig. 10, for which $r_{100}$ is $(41 \pm 8) \%$ and $(63 \pm 7) \%$, respectively. The contribution by the metallic particles is higher for the diffuse region since its SED is flatter at lower frequencies than that of the H II region (Sect. 5). The fraction obtained here is within the range of plausible values for magnetic dipole emission within the model of Draine \& Hensley (2013), and smaller than that fitted for the SMC (Draine \& Hensley 2012). In a similar analysis performed at high Galactic latitudes, Planck Collaboration Int. XVII (2014) find $r_{100}=26 \pm 6 \%$. The lower ratio follows the lower difference between their mean values for the FIR and millimetre spectral indices, $\beta_{\mathrm{FIR}}=1.65$ and $\beta_{\mathrm{mm}}=1.53$.

\subsection{Correlation between $\beta_{\mathrm{mm}}$ and $\tau_{353}$}

In this section we attempt to provide a phenomenological interpretation of the empirical correlation detected between $\beta_{\mathrm{mm}}$ and $\tau_{353}$. As mentioned in Sect. 5.2, the dust optical depth provides a measure of the quantity of matter along the line of sight, which may be atomic or molecular and which has the contribution of both dense and diffuse media. We suggest that this variation of $\beta_{\mathrm{mm}}$ with the dust optical depth can be translated into an evolution with the fraction of molecular gas along the line of sight.
The fraction of molecular gas is given by $f_{\mathrm{H}_{2}}=2 N_{\mathrm{H}_{2}} / N_{\mathrm{H}}^{\text {tot }}$. The column density of molecular hydrogen can be estimated by using the conversion factor $X_{\mathrm{CO}}=N_{\mathrm{H}_{2}} / I_{\mathrm{CO}}$, where $I_{\mathrm{CO}}$ is the ${ }^{12} \mathrm{CO} J=1 \rightarrow 0$ integrated line intensity. The Galactic $X_{\mathrm{CO}}$ conversion factor has been estimated in a variety of ways, including the use of optically thin tracers of column density such as dust emission, molecular and atomic lines, as well as using $\gamma$-ray emission. Bolatto et al. (2013) give $X_{\mathrm{CO}}=2.0 \times$ $10^{20} \mathrm{~cm}^{-2}\left(\mathrm{~K} \mathrm{~km} \mathrm{~s}^{-1}\right)^{-1}$, with $30 \%$ uncertainty, as the recommended value to use in Galactic studies. We can obtain an estimate of $X_{\mathrm{CO}}$ with the present data using the dust optical depth and the CO emission provided by the MILCA map. For that we need to include the dust specific opacity, or absorption cross-section per unit gas mass, of the molecular gas after removing the contribution of the atomic gas to the dust optical depth. Dust properties are known to evolve from the diffuse ISM to the higher density environment of molecular clouds, giving rise to an enhancement of the dust specific opacity (Planck Collaboration XXIV 2011; Planck Collaboration XXV 2011; Planck Collaboration XI 2014). One possible explanation is grain coagulation (Stepnik et al. 2003; Köhler et al. 2012). The dust specific opacity appears to be a factor of 1.5-2 times higher than the average value in the high Galactic latitude diffuse atomic ISM (Planck Collaboration XI 2014). We define the ratio between the dust opacity in the molecular and atomic media, $R$, and solve for $X_{\mathrm{CO}}$ as follows

$X_{\mathrm{CO}}=\frac{\tau_{353}}{I_{\mathrm{CO}}} \frac{N_{\mathrm{HI}}}{2 \tau_{353}} \frac{1}{R}$

where $\tau_{353} / N_{\mathrm{HI}}=\sigma_{\mathrm{HI}}=7 \times 10^{-27} \mathrm{~cm}^{2} \mathrm{H}^{-1}$ (Planck Collaboration XI 2014; Planck Collaboration Int. XVII 2014) and $\sigma_{\mathrm{H}_{2}}=R \sigma_{\mathrm{HI}}$. We remove the contribution of the atomic medium to the dust optical depth using the $N_{\mathrm{HI}}$ data from the GASS survey (Sect. 3.3) and the above value of $\sigma_{\mathrm{HI}}$. The GASS data only cover a fraction of the region under study, $l=$ $20^{\circ}-36.5$ at $b=0^{\circ}$, which is nevertheless sufficient to derive the correlation between $\mathrm{CO}$ emission and dust optical depth.

The distribution of $I_{\mathrm{CO}}$ as a function of the H I-corrected $\tau_{353}$ is shown in Fig. 11. A linear fit to the data passing through the origin, combined with Eq. (3), gives $X_{\mathrm{CO}}=1.7 \times$ $10^{20} \times(2 / R) \mathrm{cm}^{-2}\left(\mathrm{~K} \mathrm{~km} \mathrm{~s}^{-1}\right)^{-1}$. The uncertainty on this value is of $13 \%$, estimated from the scatter of the points. In order to assess the effect of a possible underestimation of the true column density of the atomic gas (Sect. 3.3), we scale $N_{\mathrm{H} \text { I }}$ by a factor of 1.5 and repeat the analysis. We find that the uncertainties on the $N_{\mathrm{HI}}$ template do not affect $X_{\mathrm{CO}}$ by more than $\sim 9 \%$. We note that the MILCA CO data in this region of the Galactic plane are about $25 \%$ higher than the $\mathrm{CO}$ data from Dame et al. (2001) (Sect. 2). Since the $X_{\mathrm{CO}}$ values in the literature refer to the Dame et al. data, we scale our result by $25 \%$ which gives $X_{\mathrm{CO}}=2.1 \times 10^{20} \times(2 / R) \mathrm{cm}^{-2}\left(\mathrm{~K} \mathrm{~km} \mathrm{~s}^{-1}\right)^{-1}$. If we assume $R=2$ then we obtain $X_{\mathrm{CO}}=X_{\mathrm{CO}}^{\mathrm{ref}}=2.1 \times 10^{20} \mathrm{~cm}^{-2}\left(\mathrm{~K} \mathrm{~km} \mathrm{~s}^{-1}\right)^{-1}$, which is consistent with the recommended value for the Galaxy given by Bolatto et al. (2013).

We can write the fraction of molecular gas along the line of sight as a function of $X_{\mathrm{CO}}$, or $R$, as

$$
f_{\mathrm{H}_{2}}=\frac{2 I_{\mathrm{CO}} X_{\mathrm{CO}}^{\mathrm{ref}}(2 / R)}{\tau_{353} / \sigma_{\mathrm{HI}}+2 I_{\mathrm{CO}} X_{\mathrm{CO}}^{\mathrm{ref}}(2 / R)(1-R)} .
$$

The correlation between $\beta_{\mathrm{mm}}$ and $f_{\mathrm{H}_{2}}$ for $R=2$ is shown in Fig. $12 \mathrm{a}$, where $\beta_{\mathrm{mm}}$ is seen to increase from atomic to molecular dominated regions. A linear fit to the data gives $\beta_{\mathrm{mm}}=$ $(1.54 \pm 0.01)+(0.12 \pm 0.01) f_{\mathrm{H}_{2}}$, meaning that $\beta_{\mathrm{mm}}=1.54$ 


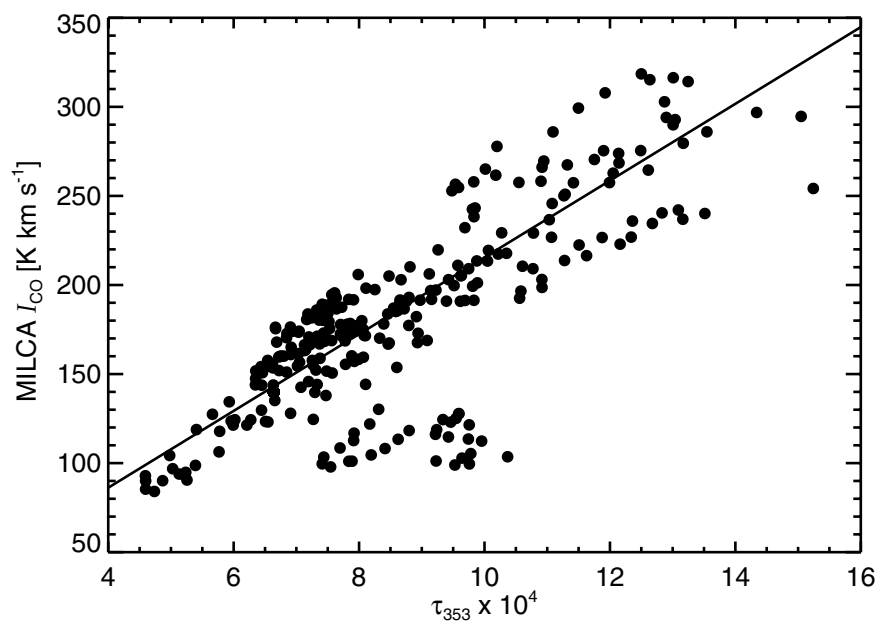

Fig. 11. The MILCA CO line intensity as a function of the dust optical depth $\tau_{353}$, corrected for the atomic gas contribution, along $b=0^{\circ}$ in the $l=20^{\circ}-36.5$ region. The line represents the linear fit to the points, from which the conversion factor $X_{\mathrm{CO}}=2.1 \times 10^{20} \times(2 / R) \mathrm{cm}^{-2}\left(\mathrm{~K} \mathrm{~km} \mathrm{~s}^{-1}\right)^{-1}$ is derived (see text).

and 1.66 at low and high values of $f_{\mathrm{H}_{2}}$, respectively. If we assume $R=1.5$ then $X_{\mathrm{CO}}=2.8 \times 10^{20} \mathrm{~cm}^{-2}\left(\mathrm{~K} \mathrm{~km} \mathrm{~s}^{-1}\right)^{-1}$, which is somewhat higher than the typical values for the Galaxy (Bolatto et al. 2013). Nevertheless, the correlation between $\beta_{\mathrm{mm}}$ and $f_{\mathrm{H}_{2}}, \beta_{\mathrm{mm}}=(1.53 \pm 0.01)+(0.13 \pm 0.01) f_{\mathrm{H}_{2}}$ is essentially unchanged. We note that we have assumed a single $T_{\mathrm{d}}$ value in the SED fit, ignoring the fact that $T_{\mathrm{d}}$ is likely to be systematically lower in molecular clouds than in the diffuse atomic medium (Planck Collaboration XIX 2011; Planck Collaboration XI 2014). However, since $T_{\mathrm{d}}$ and $\beta_{\mathrm{mm}}$ are anticorrelated, using a lower $T_{\mathrm{d}}$ in the fit would result in an even higher $\beta_{\mathrm{mm}}$ in molecular media. This would thus increase the difference in $\beta_{\mathrm{mm}}$ between atomic and molecular dominated regions. We also note that unphysical values of $f_{\mathrm{H}_{2}}$ greater than one can be reached due to data noise as well as to the assumed values of $\sigma_{\mathrm{HI}}$ and $R$. In Fig. 12, where $R=2$ and $\sigma_{\mathrm{HI}}=7 \times 10^{-27} \mathrm{~cm}^{2} \mathrm{H}^{-1}$, only $4 \%$ of the points have $f_{\mathrm{H}_{2}}>1$.

Recent results from Tabatabaei et al. (2014) show an increase of the opacity spectral index, $\beta_{\mathrm{FIR}}$, from the outer to the inner disk of M33. In addition, they find this trend to be associated with tracers of star formation and molecular gas. Their analysis is based on data between 70 and $500 \mu \mathrm{m}$, which they fit with a single and a double, cold and warm, component model. The results on $\beta_{\text {FIR }}$ from both models are consistent. We find that $\beta_{\text {FIR }}$ is also linearly correlated with $f_{\mathrm{H}_{2}}$ in the Galactic plane, as shown in Fig. 12b. A linear fit to the data gives $\beta_{\mathrm{FIR}}=(1.75 \pm 0.01)+(0.23 \pm 0.01) f_{\mathrm{H}_{2}}$, which translates into an increase of $\beta_{\text {FIR }}$ from 1.75 in atomic medium to 1.98 in molecular medium. This result compares with that found in M33, even if the wavelength range covered in our analysis does not allow the comprisal of two dust components in the fit. The same trend is found by Draine et al. (2014) in M31, where $\beta_{\text {FIR }}$ increases from $\sim 2.0$ at a distance of $15 \mathrm{kpc}$ from the centre of the galaxy to $\sim 2.3$ at $3 \mathrm{kpc}$.

\subsection{Dust evolution}

The flattening of the dust emission towards millimetre wavelengths in the plane of the Galaxy found here, is accompanied by the results of Planck Collaboration Int. XVII (2014), where the
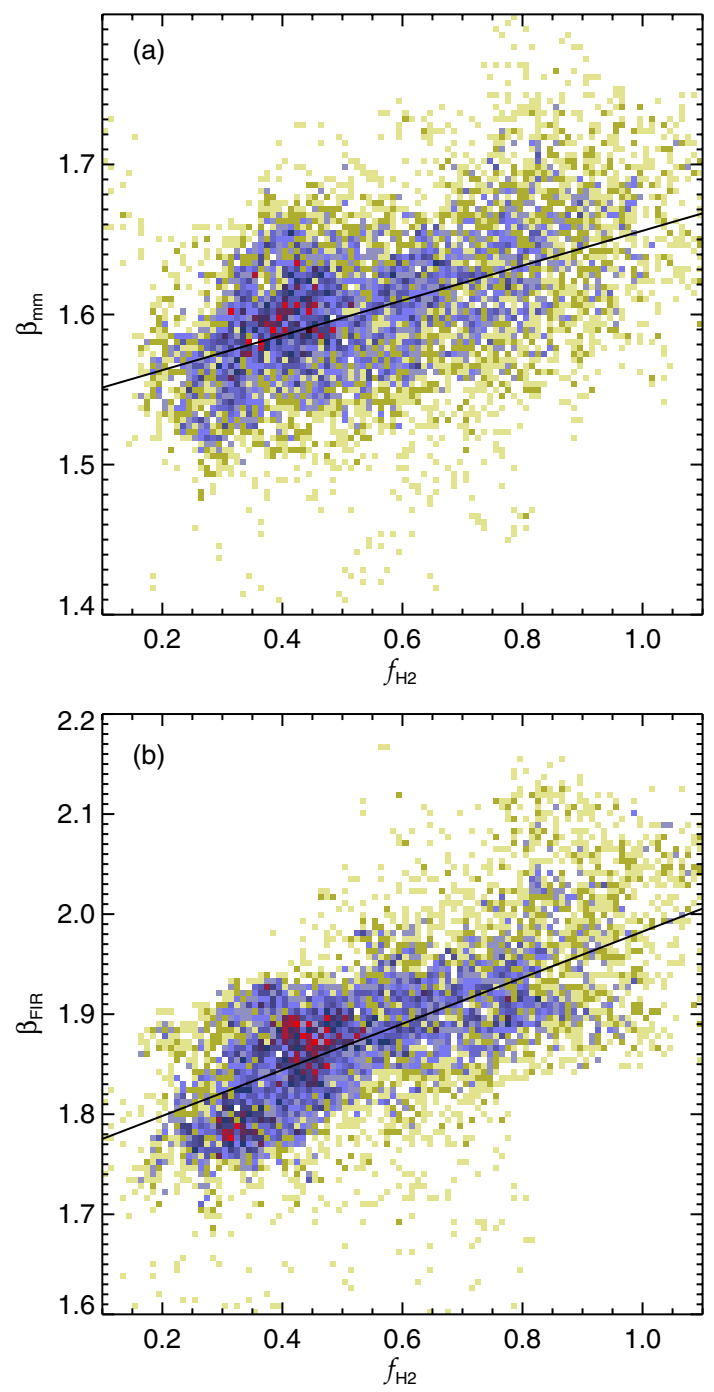

Fig. 12. Opacity spectral indices as a function of the fraction of molecular gas along the line of sight. a) $\beta_{\mathrm{mm}}$ versus $f_{\mathrm{H}_{2}}$. b) $\beta_{\mathrm{FIR}}$ versus $f_{\mathrm{H}_{2}}$. The points correspond to the thin Galactic disk, $|b| \lesssim 1^{\circ}$ or $\tau_{353} \geq 4 \times 10^{-4}$. Here $f_{\mathrm{H}_{2}}$ is estimated assuming that the dust opacity in the molecular phase is twice that of the atomic medium, $R=2$, and using $X_{\mathrm{CO}}=2.1 \times 10^{20} \mathrm{~cm}^{-2}\left(\mathrm{~K} \mathrm{~km} \mathrm{~s}^{-1}\right)^{-1}$.

same phenomenon is detected at high Galactic latitudes. In the present work $\beta_{\mathrm{FIR}}-\beta_{\mathrm{mm}} \sim 0.2$ for the atomic ISM, comparable to the value measured at high Galactic latitudes. One possibility to explain the observed change in spectral index is an increasing contribution from carbon dust at millimetre wavelengths. In the model of Jones et al. (2013) the spectral index of the carbon dust emission at $1 \mathrm{~mm}$ depends on the degree of hydrogenation and aromaticity of the grains.

It is not clear if the change of spectral index $\beta_{\mathrm{mm}}$ from atomic to molecular media is related to the observed variation of the dust specific opacity (Planck Collaboration XI 2014). Grain coagulation is a possible interpretation of the change in dust opacity. Coagulation models indicate that dust aggregation produces an overall increase of the dust specific opacity in molecular clouds, without significantly changing the apparent $\beta_{\mathrm{mm}}$ (Köhler et al. 2012). Further, there is evidence of variations in the dust opacity within the local atomic ISM (Planck Collaboration XXIV 2011; Planck Collaboration Int. XVII 2014), where grain coagulation is unlikely to occur. 
Further studies are needed to explain the correlation with the molecular material observed here, which remains phenomenological and whose origin does not rely on a physical model. In particular, Planck polarization data will be a first test of the nature of the dust SED flattening.

\section{Conclusions}

We have used Planck HFI data to derive the power-law index of the interstellar dust opacity in the frequency range 100 to $353 \mathrm{GHz}$, in a $24^{\circ}(l) \times 8^{\circ}(\mathrm{b})$ region of the Galactic plane. This is possible to achieve after the removal of the free-free emission contribution at these frequencies, which can be as high as $20-40 \%$ of the total emission in the thin and ionized disk of the Galaxy. Here we summarize our results:

- The spectral index of the dust opacity in the millimetre wavelength range, $\beta_{\mathrm{mm}}$, and in the Galactic plane has a median value of $1.60 \pm 0.06$. Thus $\beta_{\mathrm{mm}}$ is smaller than that at FIR frequencies, $\beta_{\text {FIR }}$, for which we determine a median value of $1.88 \pm 0.08$.

- We find that there is no apparent trend of $\beta_{\mathrm{mm}}$ with temperature, as opposed to $\beta_{\mathrm{FIR}}$, for which the anti-correlation has been examined in several previous studies.

- We find that $\beta_{\mathrm{mm}}$ is, however, correlated with the derived dust optical depth at $353 \mathrm{GHz}$. We interpret this correlation as an evolution of $\beta_{\mathrm{mm}}$ with the fraction of molecular gas along the line of sight, $f_{\mathrm{H}_{2}}$. Within this scenario, $\beta_{\mathrm{mm}} \sim 1.54$ when the medium is mostly atomic, whereas it increases to about 1.66 when the medium is predominantly molecular.

- The results on $\beta_{\mathrm{mm}}$ are compared with predictions from two different physical models, TLS and emission by ferromagnetic grains, which have been suggested to explain the flattening of the dust emission observed at long wavelengths. We find that both models can in principle explain the results. The same applies to the standard, two dust component models, such as DL07 and DustEM.

These results are important for understanding the dust emission from FIR to millimetre wavelengths. They are key for Galactic component separation, in particular for determining the spectral shape of the AME at high frequencies. Knowledge of the dust spectrum is also critical for estimates of the free-free emission from microwave CMB data.

Acknowledgements. We acknowledge the use of the HEALPix (Górski et al. 2005) package and IRAS data. The Planck Collaboration acknowledges support from: ESA; CNES and CNRS/INSU-IN2P3-INP (France); ASI, CNR, and INAF (Italy); NASA and DoE (USA); STFC and UKSA (UK); CSIC, MICINN and JA (Spain); Tekes, AoF and CSC (Finland); DLR and MPG (Germany); CSA (Canada); DTU Space (Denmark); SER/SSO (Switzerland); RCN (Norway); SFI (Ireland); FCT/MCTES (Portugal); and DEISA (EU). A detailed description of the Planck Collaboration and a list of its members can be found at http://www.rssd.esa.int/index.php?project=PLANCK\& page=Planck_Collaboration. The research leading to these results has received funding from the European Research Council under the European Union's Seventh Framework Programme (FP7/2007-2013)/ERC grant agreement $n^{\circ} 267934$

\section{References}

Agladze, N. I., Sievers, A. J., Jones, S. A., Burlitch, J. M., \& Beckwith, S. V. W. 1996, ApJ, 462, 1026

Ali-Haïmoud, Y., Hirata, C. M., \& Dickinson, C. 2009, MNRAS, 395, 1055 Alves, M. I. R., Davies, R. D., Dickinson, C., et al. 2010, MNRAS, 405, 1654 Alves, M. I. R., Davies, R. D., Dickinson, C., et al. 2012, MNRAS, 422, 2429 Beichman, C. A., Neugebauer, G., Habing, H. J., Clegg, P. E., \& Chester, T. J. 1988, Infrared astronomical satellite (IRAS) catalogs and atlases, Explanatory supplement, 1
Bennett, C. L., Larson, D., Weiland, J. L., et al. 2013, ApJS, 208, 20 Bersanelli, M., Mandolesi, N., Butler, R. C., et al. 2010, A\&A, 520, A4 Bolatto, A. D., Wolfire, M., \& Leroy, A. K. 2013, ARA\&A, 51, 207

Bot, C., Ysard, N., Paradis, D., et al. 2010, A\&A, 523, A20

Boudet, N., Mutschke, H., Nayral, C., et al. 2005, ApJ, 633, 272

Compiègne, M., Verstraete, L., Jones, A., et al. 2011, A\&A, 525, A103

Coupeaud, A., Demyk, K., Meny, C., et al. 2011, A\&A, 535, A124

Dame, T. M., Hartmann, D., \& Thaddeus, P. 2001, ApJ, 547, 792

Davies, R. D., Watson, R. A., \& Gutierrez, C. M. 1996, MNRAS, 278, 925

de Oliveira-Costa, A., Kogut, A., Devlin, M. J., et al. 1997, ApJ, 482, L17

Désert, F.-X., Macías-Pérez, J. F., Mayet, F., et al. 2008, A\&A, 481, 411

Dickinson, C., Davies, R. D., \& Davis, R. J. 2003, MNRAS, 341, 369

Draine, B. T. 2011, Physics of the Interstellar and Intergalactic Medium (Princeton University Press)

Draine, B. T., \& Hensley, B. 2012, ApJ, 757, 103

Draine, B. T., \& Hensley, B. 2013, ApJ, 765, 159

Draine, B. T., \& Lazarian, A. 1998, ApJ, 494, L19

Draine, B. T., \& Li, A. 2007, ApJ, 657, 810

Draine, B. T., Aniano, G., Krause, O., et al. 2014, ApJ, 780, 172

Dupac, X., Bernard, J.-P., Boudet, N., et al. 2003, A\&A, 404, L11

Finkbeiner, D. P. 2003, ApJS, 146, 407

Finkbeiner, D. P., Davis, M., \& Schlegel, D. J. 1999, ApJ, 524, 867

Galliano, F., Madden, S. C., Jones, A. P., Wilson, C. D., \& Bernard, J.-P. 2005, A\&A, 434, 867

Galliano, F., Hony, S., Bernard, J.-P., et al. 2011, A\&A, 536, A88

Ghosh, T., Banday, A. J., Jaffe, T., et al. 2012, MNRAS, 422, 3617

Gordon, M. A., \& Sorochenko, R. L. 2009, Radio Recombination Lines (Springer Verlag), Astrophys. Space Sci. Lib.

Gordon, K. D., Galliano, F., Hony, S., et al. 2010, A\&A, 518, L89

Górski, K. M., Hivon, E., Banday, A. J., et al. 2005, ApJ, 622, 759

Hoang, T., Draine, B. T., \& Lazarian, A. 2010, ApJ, 715, 1462

Hoang, T., Lazarian, A., \& Draine, B. T. 2011, ApJ, 741, 87

Hurier, G., Macías-Pérez, J. F., \& Hildebrandt, S. 2013, A\&A, 558, A118

Israel, F. P., Wall, W. F., Raban, D., et al. 2010, A\&A, 519, A67

Jones, A. P., Fanciullo, L., Köhler, M., et al. 2013, A\&A, 558, A62

Juvela, M., \& Ysard, N. 2012a, A\&A, 541, A33

Juvela, M., \& Ysard, N. 2012b, A\&A, 539, A71

Kalberla, P. M. W., McClure-Griffiths, N. M., Pisano, D. J., et al. 2010, A\&A, 521, A17

Kelly, B. C., Shetty, R., Stutz, A. M., et al. 2012, ApJ, 752, 55

Kogut, A., Banday, A. J., Bennett, C. L., et al. 1996, ApJ, 464, L5

Köhler, M., Stepnik, B., Jones, A. P., et al. 2012, A\&A, 548, A61

Lagache, G., Bavouzet, N., Fernandez-Conde, N., et al. 2007, ApJ, 665, L89

Lamarre, J., Puget, J., Ade, P. A. R., et al. 2010, A\&A, 520, A9

Leahy, J. P., Bersanelli, M., D’Arcangelo, O., et al. 2010, A\&A, 520, A8

Leitch, E. M., Readhead, A. C. S., Pearson, T. J., \& Myers, S. T. 1997, ApJ, 486, L23

Mandolesi, N., Bersanelli, M., Butler, R. C., et al. 2010, A\&A, 520, A3

Markwardt, C. B. 2009, in Astronomical Data Analysis Software and Systems XVIII, eds. D. A. Bohlender, D. Durand, \& P. Dowler, ASP Conf. Ser., 411, 251

Mathis, J. S., Mezger, P. G., \& Panagia, N. 1983, A\&A, 128, 212

McClure-Griffiths, N. M., Pisano, D. J., Calabretta, M. R., et al. 2009, ApJS, 181,398

Mennella, A., Butler, R. C., Curto, A., et al. 2011, A\&A, 536, A3

Meny, C., Gromov, V., Boudet, N., et al. 2007, A\&A, 468, 171

Miville-Deschênes, M., \& Lagache, G. 2005, ApJS, 157, 302

Miville-Deschênes, M.-A., Lagache, G., \& Puget, J.-L. 2002, A\&A, 393, 749

Paladini, R., Montier, L., Giard, M., et al. 2007, A\&A, 465, 839

Paradis, D., Bernard, J.-P., \& Mény, C. 2009, A\&A, 506, 745

Paradis, D., Veneziani, M., Noriega-Crespo, A., et al. 2010, A\&A, 520, L8

Paradis, D., Bernard, J.-P., Mény, C., \& Gromov, V. 2011, A\&A, 534, A118

Paradis, D., Paladini, R., Noriega-Crespo, A., et al. 2012, A\&A, 537, A113

Planck Collaboration I. 2011, A\&A, 536, A1

Planck Collaboration XVII. 2011, A\&A, 536, A17

Planck Collaboration XVIII. 2011, A\&A, 536, A18

Planck Collaboration XIX. 2011, A\&A, 536, A19

Planck Collaboration XX. 2011, A\&A, 536, A20

Planck Collaboration XXIV. 2011, A\&A, 536, A24

Planck Collaboration XXV. 2011, A\&A, 536, A25

Planck Collaboration Int. XII. 2013, A\&A, 557, A53

Planck Collaboration II. 2014, A\&A, in press, DOI: 10.1051/0004-6361/201321550

Planck Collaboration VI. 2014, A\&A, submitted [arXiv: 1303. 5067]

Planck Collaboration IX. 2014, A\&A, in press, DOI: 10.1051/0004-6361/201321531

Planck Collaboration XI. 2014, A\&A, submitted 
Planck Collaboration XII. 2014, A\&A, in press, DOI: $10.1051 / 0004-6361 / 201321580$

Planck Collaboration XIII. 2014, A\&A, submitted [arXiv: 1303.5073]

Planck Collaboration XIV. 2014, A\&A, in press, DOI: $10.1051 / 0004-6361 / 201321562$

Planck Collaboration Int. XV. 2014, A\&A, in press [arXiv: 1309.1357]

Planck Collaboration Int. XVII. 2014, A\&A, submitted DOI:10.1051/00046361/201323270

Planck HFI Core Team. 2011, A\&A, 536, A4

Reach, W. T., Dwek, E., Fixsen, D. J., et al. 1995, ApJ, 451, 188

Rohlfs, K., \& Wilson, T. L. 2000, Tools of Radio Astronomy (New York: Springer)

Rosset, C., Tristram, M., Ponthieu, N., et al. 2010, A\&A, 520, A13

Sajina, A., Scott, D., Dennefeld, M., et al. 2006, MNRAS, 369, 939

Shetty, R., Kauffmann, J., Schnee, S., \& Goodman, A. A. 2009a, ApJ, 696, 676

Shetty, R., Kauffmann, J., Schnee, S., Goodman, A. A., \& Ercolano, B. 2009b, ApJ, 696, 2234

Spitzer, L. 1978, Physical processes in the interstellar medium (New York: Wiley)

Staveley-Smith, L., Wilson, W. E., Bird, T. S., et al. 1996, Publ. Astron. Soc. Aust., 13, 243

Staveley-Smith, L., Juraszek, S., Koribalski, B. S., et al. 1998, AJ, 116, 2717

Stepnik, B., Abergel, A., Bernard, J.-P., et al. 2003, A\&A, 398, 551

Strasser, S., \& Taylor, A. R. 2004, ApJ, 603, 560

Tabatabaei, F. S., Braine, J., Xilouris, E. M., et al. 2014, A\&A, 561, A95

Tauber, J. A., Mandolesi, N., Puget, J., et al. 2010, A\&A, 520, A1

Ysard, N., \& Verstraete, L. 2010, A\&A, 509, A12

1 APC, AstroParticule et Cosmologie, Université Paris Diderot, CNRS/IN2P3, CEA/lrfu, Observatoire de Paris, Sorbonne Paris Cité, 10 rue Alice Domon et Léonie Duquet, 75205 Paris Cedex 13, France

2 Aalto University Metsähovi Radio Observatory and Dept of Radio Science and Engineering, PO Box 13000, 00076 Aalto, Finland

3 African Institute for Mathematical Sciences, 6-8 Melrose road, Muizenberg, Cape Town, 7701 Rondebosch, South Africa

4 Agenzia Spaziale Italiana Science Data Center, via del Politecnico snc, 00133 Roma, Italy

5 Agenzia Spaziale Italiana, viale Liegi 26, 00198 Roma, Italy

6 Astrophysics Group, Cavendish Laboratory, University of Cambridge, J J Thomson avenue, Cambridge CB3 0HE, UK

7 Astrophysics \& Cosmology Research Unit, School of Mathematics, Statistics \& Computer Science, University of KwaZulu-Natal, Westville Campus, Private Bag X54001, 4000 Durban, South Africa

8 Atacama Large Millimeter/submillimeter Array, ALMA Santiago Central Offices, Alonso de Córdova 3107, Vitacura, Casilla 763 0355, Santiago, Chile

9 CITA, University of Toronto, 60 St. George St., Toronto ON M5S 3H8, Canada

10 CNRS, IRAP, 9 Av. colonel Roche, BP 44346, 31028 Toulouse Cedex 4, France

11 California Institute of Technology, Pasadena, USA

12 Centro de Estudios de Física del Cosmos de Aragón (CEFCA), plaza San Juan 1, planta 2, 44001 Teruel, Spain

13 Computational Cosmology Center, Lawrence Berkeley National Laboratory, Berkeley, USA

14 Consejo Superior de Investigaciones Científicas (CSIC), 28037 Madrid, Spain

15 DSM/Irfu/SPP, CEA-Saclay, 91191 Gif-sur-Yvette Cedex, France

16 DTU Space, National Space Institute, Technical University of Denmark, Elektrovej 327, 2800 Kgs. Lyngby, Denmark

17 Département de Physique Théorique, Université de Genève, 24 Quai E. Ansermet, 1211 Genève 4, Switzerland

18 Departamento de Física Fundamental, Facultad de Ciencias, Universidad de Salamanca, 37008 Salamanca, Spain

19 Departamento de Física, Universidad de Oviedo, Avda. Calvo Sotelo s/n, 33007 Oviedo, Spain
20 Department of Astrophysics/IMAPP, Radboud University Nijmegen, PO Box 9010, 6500 GL Nijmegen, The Netherlands

21 Department of Electrical Engineering and Computer Sciences, University of California, Berkeley, USA

22 Department of Physics \& Astronomy, University of British Columbia, 6224 Agricultural road, Vancouver, Canada

23 Department of Physics and Astronomy, Dana and David Dornsife College of Letter, Arts and Sciences, University of Southern California, Los Angeles CA 90089, USA

24 Department of Physics and Astronomy, University College London, London WC1E 6BT, UK

25 Department of Physics, Gustaf Hällströmin katu 2a, University of Helsinki, Helsinki, Finland

${ }^{26}$ Department of Physics, Princeton University, Princeton, USA

27 Department of Physics, University of California, Santa Barbara, USA

28 Department of Physics, University of Illinois at UrbanaChampaign, 1110 West Green street, Urbana, USA

29 Dipartimento di Fisica e Astronomia G. Galilei, Università degli Studi di Padova, via Marzolo 8, 35131 Padova, Italy

30 Dipartimento di Fisica e Scienze della Terra, Università di Ferrara, via Saragat 1, 44122 Ferrara, Italy

31 Dipartimento di Fisica, Università La Sapienza, P. le A. Moro 2, 00185 Roma, Italy

32 Dipartimento di Fisica, Università degli Studi di Milano, via Celoria 16, 20133 Milano, Italy

33 Dipartimento di Fisica, Università degli Studi di Trieste, via A. Valerio 2, 34127 Trieste, Italy

34 Dipartimento di Fisica, Università di Roma Tor Vergata, via della Ricerca Scientifica 1, 00133 Roma, Italy

35 Discovery Center, Niels Bohr Institute, Blegdamsvej 17, 2100 Copenhagen, Denmark

36 Dpto. Astrofísica, Universidad de La Laguna (ULL), 38206 La Laguna, Tenerife, Spain

37 European Southern Observatory, ESO Vitacura, Alonso de Córdova 3107, Vitacura, Casilla 19001, Santiago, Chile

38 European Space Agency, ESAC, Planck Science Office, Camino bajo del Castillo s/n, Urbanización Villafranca del Castillo, Villanueva de la Cañada, Madrid, Spain

39 European Space Agency, ESTEC, Keplerlaan 1, 2201 AZ Noordwijk, The Netherlands

40 Helsinki Institute of Physics, Gustaf Hällströmin katu 2, University of Helsinki, 00014 Helsinki, Finland

41 INAF - Osservatorio Astronomico di Padova, vicolo dell'Osservatorio 5, 35122 Padova, Italy

42 INAF - Osservatorio Astronomico di Roma, via di Frascati 33, 00040 Monte Porzio Catone, Italy

43 INAF - Osservatorio Astronomico di Trieste, via G.B. Tiepolo 11, 34143 Trieste, Italy

44 INAF/IASF Bologna, via Gobetti 101, 40129 Bologna, Italy

45 INAF/IASF Milano, via E. Bassini 15, 20133 Milano, Italy

46 INFN, Sezione di Bologna, via Irnerio 46, 40126 Bologna, Italy

47 INFN, Sezione di Roma 1, Università di Roma Sapienza, piazzale Aldo Moro 2, 00185 Roma, Italy

48 INFN/National Institute for Nuclear Physics, via Valerio 2, 34127 Trieste, Italy

49 IPAG: Institut de Planétologie et d'Astrophysique de Grenoble, Université Joseph Fourier, Grenoble 1/CNRS-INSU, UMR 5274, 38041 Grenoble, France

50 IUCAA, Post Bag 4, Ganeshkhind, Pune University Campus, 411007 Pune, India

51 Imperial College London, Astrophysics group, Blackett Laboratory, Prince Consort road, London SW7 2AZ, UK

52 Infrared Processing and Analysis Center, California Institute of Technology, Pasadena CA 91125, USA

53 Institut Universitaire de France, 103 Bd Saint-Michel, 75005 Paris, France 
54 Institut d'Astrophysique Spatiale, CNRS (UMR8617) Université Paris-Sud 11, Bâtiment 121, 91405 Orsay, France

55 Institut d'Astrophysique de Paris, CNRS (UMR7095), 98 bis boulevard Arago, 75014 Paris, France

56 Institute for Space Sciences, 077125 Bucharest-Magurale, Romania

57 Institute of Astronomy and Astrophysics, Academia Sinica, 10617 Taipei, Taiwan

58 Institute of Astronomy, University of Cambridge, Madingley Road, Cambridge CB3 0HA, UK

59 Institute of Theoretical Astrophysics, University of Oslo, Blindern, 0315 Oslo, Norway

${ }^{60}$ Instituto de Astrofísica de Canarias, C/vía Láctea s/n, 38205 La Laguna, Tenerife, Spain

61 Instituto de Física de Cantabria (CSIC-Universidad de Cantabria), Avda. de los Castros s/n, 39005 Santander, Spain

62 Jet Propulsion Laboratory, California Institute of Technology, 4800 Oak Grove drive, Pasadena, USA

63 Jodrell Bank Centre for Astrophysics, Alan Turing Building, School of Physics and Astronomy, The University of Manchester, Oxford Road, Manchester M13 9PL, UK

${ }^{64}$ Kavli Institute for Cosmology Cambridge, Madingley Road, Cambridge CB3 0HA, UK

65 LAL, Université Paris-Sud, CNRS/IN2P3, Orsay, France

66 LERMA, CNRS, Observatoire de Paris, 61 avenue de l'Observatoire, 75014 Paris, France

67 Laboratoire AIM, IRFU/Service d'Astrophysique - CEA/DSM CNRS - Université Paris Diderot, Bât. 709, CEA-Saclay, 91191 Gif-sur-Yvette Cedex, France

68 Laboratoire Traitement et Communication de l'Information, CNRS (UMR 5141) and Télécom ParisTech, 46 rue Barrault, 75634 Paris Cedex 13, France

${ }^{69}$ Laboratoire de Physique Subatomique et de Cosmologie, Université Joseph Fourier Grenoble I, CNRS/IN2P3, Institut National Polytechnique de Grenoble, 53 rue des Martyrs, 38026 Grenoble Cedex, France
70 Laboratoire de Physique Théorique, Université Paris-Sud 11 \& CNRS, Bâtiment 210, 91405 Orsay, France

${ }^{71}$ Lawrence Berkeley National Laboratory, Berkeley, USA

72 Max-Planck-Institut für Astrophysik, Karl-Schwarzschild-Str. 1, 85741 Garching, Germany

73 National University of Ireland, Department of Experimental Physics, Maynooth, Co. Kildare, Ireland

74 Niels Bohr Institute, Blegdamsvej 17, Copenhagen, Denmark

75 Observational Cosmology, Mail Stop 367-17, California Institute of Technology, Pasadena CA 91125, USA

76 Optical Science Laboratory, University College London, Gower Street, London, UK

77 SISSA, Astrophysics Sector, via Bonomea 265, 34136 Trieste, Italy

78 School of Physics and Astronomy, Cardiff University, Queens Buildings, The Parade, Cardiff CF24 3AA, UK

79 Space Sciences Laboratory, University of California, Berkeley, California, USA

80 Special Astrophysical Observatory, Russian Academy of Sciences, Nizhnij Arkhyz, Zelenchukskiy region, 369167 KarachaiCherkessian Republic, Russia

81 UPMC Univ Paris 06, UMR7095, 98 bis boulevard Arago, 75014 Paris, France

82 Université de Toulouse, UPS-OMP, IRAP, 31028 Toulouse Cedex 4, France

83 Universities Space Research Association, Stratospheric Observatory for Infrared Astronomy, MS 232-11, Moffett Field CA 94035, USA

${ }^{84}$ University of Granada, Departamento de Física Teórica y del Cosmos, Facultad de Ciencias, Granada, Spain

85 Warsaw University Observatory, Aleje Ujazdowskie 4, 00-478 Warszawa, Poland 